\title{
Transonic Aerodynamic Loads Modeling of X-31 Aircraft
}

\author{
Mehdi Ghoreyshi, Martiqua L. Post $†$ and Russell M. Cummings ${ }^{\ddagger}$ \\ Modeling and Simulation Research Center, U.S. Air Force Academy \\ USAF Academy, Colorado 80840-6400 \\ Andrea Da Ronch ${ }^{\S}$ and Kenneth J. Badcock ${ }^{\mathbb{\Phi}}$ \\ CFD Lab, University of Liverpool \\ Liverpool, UK, L69 3GH
}

\begin{abstract}
The generation of reduced order models for computing the unsteady and nonlinear aerodynamic loads on the X-31 aircraft from pitching motions in the transonic speed range is described. The models considered are based on Duhamel's superposition integral using indicial (step) response functions, Volterra theory using nonlinear kernels, Radial Basis functions, and a surrogate-based recurrence framework, both using time-history simulations of a training maneuver(s). One of the biggest challenges in creating these reduced order modeling techniques is accurate identification of unknowns. A large number of step function calculation is required for any combination of angle of attack and free-stream Mach number. A method to efficiently reduce the number of step function calculations is proposed. This method uses a time-dependent surrogate model to fit the relationship between flight conditions (Mach number and angle of attack) and step functions calculated from a limited number of simulations (samples). Each sample itself is directly calculated from unsteady Reynolds-Averaged Navier-Stokes simulations starting from an initial steady-state condition with a prescribed grid motion. An indirect method is proposed to estimate the nonlinear Volterra kernels from time-accurate computational fluid dynamic simulations of different training maneuvers. These maneuvering simulations were also used to estimate the unknown parameters in a model based on Radial Basis functions. A Design of Experiment method was used to generate several pitching motions at different amplitudes and free-stream Mach numbers. The model based on a surrogate-based recurrence framework then approximates the aerodynamic responses induced by pitching motions at new flight conditions. Results are reported for the X-31 configuration with a sharp leading-edge geometry, including canard/wing vortices. The validity of models studied was assessed by comparison of the model outputs with time-accurate computational fluid dynamic simulations of new maneuvers. Overall, the reduced order models were found to produce accurate results, although a nonlinear model based on indicial functions yielded the best accuracy among all models. This model, along with a developed time-dependent surrogate approach, helped to produce accurate predictions for a wide range of motions in the transonic speed range within a few seconds.
\end{abstract}

\section{Introduction}

Modern fighter aircraft often maneuver in extreme conditions of the flight envelope characterized by vortical flows and shock effects. Such conditions are important for unsteady flow which can cause flight

\footnotetext{
${ }^{*}$ NRC Research Fellow, AIAA Senior Member

$\dagger$ Associate Professor, AIAA Senior Member

¥Professor of Aeronautics, AIAA Associate Fellow

$\S$ Research Associate, AIAA Member

IProfessor of Computational Aerodynamics, AIAA Senior Member
} 
dynamic and aeroelastic instabilities. ${ }^{1}$ The linearized unsteady aerodynamic models no longer work at these conditions. Currently, the use of computational fluid dynamics (CFD) solutions is considered as the state of the art in modeling unsteady nonlinear flow physics ${ }^{2}$ and offers an early and improved understanding and prediction of aircraft aerodynamic characteristics. It is well known that unsteady aerodynamic forces and moments not only depend on the instantaneous states but also by all of the states at times prior to the current state. ${ }^{3}$ Unfortunately, this makes the solution of the aircraft equations of motion an infinitedimensional problem, where the current states depend on the evolution of previous states at infinitely many points in time. ${ }^{4}$ With the advances in computing techniques, one straightforward way to solve this problem is to develop a full-order model based on direct solution of discretized Reynolds-Averaged Navier-Stokes (RANS) equations coupled with the dynamic equations governing the aircraft motion. ${ }^{5-7}$

Creating a full-order model for Stability \& Control (S\&C) analysis is a computationally very expensive approach since such a model needs a large number of coupled computations for different values of motion frequency and amplitude. ${ }^{8}$ An alternative approach to creating the full-order model is to develop a Reduced Order Model (ROM) that seeks to approximate CFD results by extracting information from a limited number of full-order simulations. ${ }^{9,10}$ Ideally, the specified ROM can predict aircraft responses over a wide range of amplitude and frequency within a few seconds without the need of running CFD simulations again.

Recent efforts on the development of ROMs can be classified into two types: time domain or frequency domain approaches. ${ }^{11}$ The frequency domain models are obtained from matching transfer functions computed from the measured input-output data. ${ }^{12}$ Examples of the frequency domain ROMs are the indicial response method by Ballhaus and Goorjian ${ }^{13}$ and Tobak et al., ${ }^{14,15}$ and a frequency-domain approach based on proper orthogonal decomposition by Hall. ${ }^{16}$ Some examples of time domain ROMs include the unit sample response by Gaitonde and Jones, ${ }^{17}$ Volterra theory by Silva and Bartels, ${ }^{18}$ Radial Basis Functions $(\mathrm{RBF})^{19}$ and state-space modeling. ${ }^{20}$ These ROM techniques have been used extensively for flutter prediction, limit cycle oscillation, and gust response modeling, ${ }^{21}$ but their application to S\&C is still new. Also, only a few studies have been conducted for reduced order modeling of aircraft configurations, mostly limited to the subsonic flow regime. The current paper aims to assess the accuracy of prediction and incurred computational cost of reduced order models based on the indicial response method, Volterra theory, RBF, and a Surrogate-Based Recurrence-Framework (SBRF) for X-31 aircraft pitching motions in the transonic speed range. More details about these models are provided in section II

The transient aerodynamic response due to a step change in a forcing parameter, such as angle of attack or pitch rate is a so-called "indicial function". Assuming that the indicial functions are known, the aerodynamic forces and moments induced in any maneuver can be estimated by using the well-known Duhamel superposition integral. ${ }^{22}$ Tobak et al. ${ }^{14,15}$ and Reisenthel et al. ${ }^{23,24}$ detailed the superposition process for the modeling of unsteady lift and pitch moment from angle of attack and pitch rate indicial functions. Ghoreyshi and Cummings ${ }^{25}$ extended this model to include lateral forces and moments as well. The accuracy of ROMs based on indicial functions depends, to a large extent, on accurate identification of indicial functions; these functions can be derived from analytical, CFD, or experimental methods. ${ }^{26}$ Limited analytical expressions of indicial functions exist for two-dimensional airfoils. ${ }^{27}$ For incompressible flows, Wagner $^{28}$ was the first who detailed the analytical unsteady lift of a thin airfoil undergoing a plunging motion using a single indicial function (the so-called Wagner's function) with its exact values defined in terms of Bessel functions. For unsteady, compressible flow past two-dimensional airfoils, Bisplinghoff et al. ${ }^{29}$ also described an exponential approximation to the exact solutions of the indicial functions at different Mach numbers. However, these analytical expressions are not valid for aircraft configurations due to the three-dimensional tip vortices.

Direct and indirect methods are used to estimate indicial functions. Leishman ${ }^{30}$ has presented an indirect technique for identifying indicial functions from aerodynamic responses due to harmonic motions. However, the derived indicial functions using indirect methods depend largely on the quality of motion, e.g. amplitude and frequency. Experimental tests are practically nonexistent for direct indicial function measurements due to wind tunnel constraints. An alternative is to use CFD, but special considerations are required to simulate step responses in CFD. Singh and Baeder ${ }^{31}$ used a surface transpiration approach to directly calculate the angle of attack indicial response using CFD. Ghoreyshi et al. ${ }^{32}$ also described an approach based on a grid motion technique for CFD-type calculation of linear and nonlinear inidical functions with respect to angle of attack and pitch rate. Ghoreyshi and Cummings ${ }^{25}$ later used this approach to generate longitudinal and lateral indicial functions for a generic unmanned combat air vehicle and used these functions for predicting the aerodynamic responses to aircraft six degrees of freedom maneuvers. In this paper, the transonic indicial 
functions of the X-31 aircraft are calculated using CFD and the grid motion approach.

For motions at low angles of attack, and assuming incompressible flow, only a single indicial function with respect to each forcing parameter needs to be generated. ${ }^{33}$ For flows at transonic speed, however many indicial functions need to be generated for each combination of angle of attack and free-stream Mach number. The generation of all these functions using CFD is expensive and makes the creation of a ROM very time consuming. Note that these models are still cheaper than full-order simulations because the ROMs based on indicial functions eliminate the need to repeat calculations for each frequency. A cost-effective unsteady aerodynamic model needs a mathematical description of indicial functions as a function of angle of attack and free-stream Mach number. However, this model is often unavailable for three-dimensional configurations. It is more common to use surrogate models which are mathematical approximations of the true response of the system, built using some observed responses, and therefore the total cost of creating ROM is reduced. In this paper, a surrogate model is proposed based on the Kriging technique ${ }^{34}$ to model indicial functions as a function of angle of attack and free-stream Mach number.

Volterra's functional mathematics is considered as one of the most important tools for the representation of non-linear systems where the system output depends on the current and past values of the input. The approach was first introduced by Vito Volterra in a book published in 1930. ${ }^{35}$ This work and further developments by others (for example N. Wiener, ${ }^{36}$ Barrett $^{37}$ and Kalman ${ }^{38}$ ) have been extensively used in electrical and biological systems engineering. ${ }^{39-43}$ Recently, there is an increasing interest in using Volterra theory in the field of aerodynamic loads modeling, ${ }^{2,44}$ where the aerodynamic forces and moments are approximated by an infinite series of multi-dimensional convolution integrals of the inputs and increasing order kernels named the so-called Volterra kernels. ${ }^{45}$ Generally the Volterra kernels are not known and need to be determined. Silva ${ }^{46}$ used the first and second terms of the Volterra functions and proposed a method based on the impulse functions to directly calculate first- and second-order kernels using CFD. In his approach the first-order kernel is a combination of the response to unit and double unit impulses at time $t_{1}=T$. The second-order kernel is a combination of two successive unit impulses at time $t_{1}=T$ and $t_{2}=T+\Delta t$ and two unit pulses, one at time $T$, and a second at time $T+\Delta t$. Jirasek and Cummings ${ }^{44}$ used this approach to find Volterra kernels for X-31 aircraft at subsonic speeds. However, the CFD simulation of system impulse response at transonic speeds is very complicated as the impulse occurs over a very short period of time. Da Ronch et al. ${ }^{47}$ proposed an approach of determining these functions from unsteady timedomain solutions. In this paper, the chirp and spiral training maneuvers are used to estimate the Volterra kernels.

The complex CFD system (that is flow, flow equations, and boundary conditions) can be represented by a simplified input-output relationship. The relationship can be learned using neural networks and surrogate models. ${ }^{45,48-50}$ Marques and Anderson ${ }^{45}$ used a temporal neural network to approximate the unsteady lift and pitch moment of a 2D airfoil for the changes in angle of attack in the transonic regime. Although, they found a good match for the lift, the predictions of pitch moment did not match as well. The network outputs in their work depended on time histories of angle of attack only and not previous values of lift and pitch moment. Faller and Schreck ${ }^{49}$ also used a neural network to predict the time-dependent surface pressures of a pitching wing. The network inputs included the instantaneous pitch angle, angular velocity, and the initial surface pressure coefficients at $t_{0}$. The network output then predicted the surface pressures at time $t_{0}+\Delta t$. These predictions were fed back as the input to the network to predict surface pressures at the next time interval. This process continues with time until it reaches the final time. However, this model does not have the time histories of motion variables, therefore it might not have sufficient information for modeling highly unsteady flows. Glaz et al. ${ }^{50}$ developed a mapping between aerodynamic loads and time histories of both motion parameters and loads and then tried to learn this mapping using a surrogate model with the aid of Design of Experiments. Ghoreyshi et al. ${ }^{51}$ extended this mapping to include both RANS and Euler calculations and used RBF neural networks trained from some special training maneuvers. Da Ronch et al. ${ }^{47}$ also tried to establish this mapping using a SBRF model for a pitching airfoil at transonic speed. A key issue in this modeling is the effects of the training motion on identifying model parameters. This paper discusses the effects of three training motions: chirp, spiral, and Schroeder. ${ }^{19}$

All these reduced order models require different computational costs and are based on various simplifying assumptions pertaining to the flow physics. For example, the generation of linear indicial functions are relatively inexpensive, but the model cannot accurately capture nonlinear flow phenomena associated with transonic flow and/or dynamic stall. The accuracy of models based on RBF and SBRF depends to a large extend on the training maneuvers used. Therefore in this paper, these models are tested for predicting the 
unsteady loads induced in pitching motion at transonic speeds. The configuration, the X-31 aircraft, has highly swept slender wings as shown in Fig. 1 resulting in complex vortical flow under various conditions. The unsteady aerodynamic modeling of this configuration at transonic regime is very complicated due to strongly nonlinear aerodynamic phenomena associated with shock effects and vortical flow.

The test case, geometry definition, and ROMs will first be detailed. The paper continues to describe the generation of ROMs. Finally, the accuracy of the aerodynamic models will be compared with time-dependent CFD computations of new maneuvers.

\section{Formulation}

\section{A. CFD Solver}

The flow solver used for this study is the Cobalt $\operatorname{code}^{52}$ that solves the unsteady, three-dimensional and compressible Navier-Stokes equations in an inertial reference frame. These equations in integral form are ${ }^{53}$

$$
\frac{\partial}{\partial t} \iiint \mathbf{Q} d V+\iint(\mathbf{f} \hat{i}+\mathbf{g} \hat{j}+\mathbf{h} \hat{k}) \cdot \hat{n} d S=\iint(\mathbf{r} \hat{i}+\mathbf{s} \hat{j}+\mathbf{t} \hat{k}) \cdot \hat{n} d S
$$

where $V$ is the fluid element volume; $S$ is the fluid element surface area; $\hat{n}$ is the unit normal to $S ; \hat{i}, \hat{j}$, and $\hat{k}$ are the Cartesian unit vectors; $\mathbf{Q}=(\rho, \rho u, \rho v, \rho w, \rho e)^{T}$ is the vector of conserved variables, where $\rho$ represents air density, $u, v, w$ are velocity components and $e$ is the specific energy per unit volume. The vectors of $\mathbf{f}, \mathbf{g}$, and $\mathbf{h}$ represent the inviscid components and are detailed below

$$
\begin{aligned}
\mathbf{f} & =\left(\rho u, \rho u^{2}+p, \rho u v, \rho u w, u(\rho e+p)\right)^{T} \\
\mathbf{g} & =\left(\rho v, \rho v^{2}+p, \rho v u, \rho v w, v(\rho e+p)\right)^{T} \\
\mathbf{h} & =\left(\rho w, \rho w^{2}+p, \rho w v, \rho w v, w(\rho e+p)\right)^{T}
\end{aligned}
$$

where the superscript $T$ denotes the transpose operation. The vectors of $\mathbf{r}, \mathbf{s}$, and $\mathbf{t}$ represent the viscous components are described as

$$
\begin{aligned}
& \mathbf{r}=\left(0, \tau_{x x}, \tau_{x y}, \tau_{x z}, u \tau_{x x}+v \tau_{x y}+w \tau_{x z}+k T_{x}\right)^{T} \\
& \mathbf{s}=\left(0, \tau_{x y}, \tau_{y y}, \tau_{y z}, u \tau_{x y}+v \tau_{y y}+w \tau_{y z}+k T_{y}\right)^{T} \\
& \mathbf{t}=\left(0, \tau_{x z}, \tau_{z y}, \tau_{z z}, u \tau_{x z}+v \tau_{z y}+w \tau_{z z}+k T_{z}\right)^{T}
\end{aligned}
$$

where $\tau_{i j}$ are the viscous stress tensor components; $T$ is the temperature; and $k$ is the thermal conductivity. The ideal gas law closes the system of equations and the entire equation set is nondimensionalized by freestream density and speed of sound. ${ }^{52}$ The Navier-Stokes equations are discretised on arbitrary grid topologies using a cell-centered finite volume method. Second-order accuracy in space is achieved using the exact Riemann solver of Gottlieb and Groth, ${ }^{54}$ and least squares gradient calculations using QR factorization. To accelerate the solution of discretized system, a point-implicit method using analytic first-order inviscid and viscous Jacobians. A Newtonian sub-iteration method is used to improve time accuracy of the point-implicit method. Tomaro et al. ${ }^{55}$ converted the code from explicit to implicit, enabling Courant-Friedrichs-Lewy numbers as high as $10^{6}$. The Cobalt solver has been used at the Air Force Seek Eagle Office (AFSEO) and the United States Air Force Academy (USAFA) for a variety of unsteady nonlinear aerodynamic problems of maneuvering aircraft. ${ }^{56-60}$

\section{B. ROMs Based on Volterra Theory}

The X-31 aircraft pitch moment modeling is considered in this paper, which is highly nonlinear in the transonic regime. The output function $y$ is defined as $y=C_{m}(t)$. Using Volterra theory, ${ }^{35}$ the output of a continuous-time, casual, time-invariant, fading memory system in response to an input, $x(t)$, can be modeled using the $p$-th order Volterra series:

$$
y(t)=\Psi(x(t))=\sum_{i=1}^{p} \mathbf{H}_{i}(x(t))
$$


where, $\mathbf{H}_{i}$ represents the $i$-th order Volterra operator, which is defined as an $i$-fold convolution between the input, $x(t)$, and the $i$-th order Volterra kernel, $H_{i}$, i.e.

$$
\mathbf{H}_{i}(x(t))=\int_{-\infty}^{t} \ldots \int_{-\infty}^{t} H_{i}\left(t-\tau_{1}, t-\tau_{2}, \ldots, t-\tau_{i}\right) \prod_{n=1}^{i} x\left(\tau_{n}\right) d \tau_{n}
$$

where, for forced oscillations about the pitch axis, the input vector includes:

$$
\mathbf{x}(t)=(\alpha(t), \dot{\alpha}(t), \ddot{\alpha}(t))
$$

A multi-input Volterra series is then formulated as

$$
y(t)=\Psi\left(x_{1}(t), x_{2}(t), \ldots, x_{m}(t)\right)=\sum_{i=1}^{p} \mathbf{H}_{i}^{m}
$$

The term $\mathbf{H}_{i}^{m}$ is the multi-input Volterra operator defined as a $m^{p}$-fold summation of $p$-fold convolution integrals between the inputs and the $p$-th order multi-input Volterra kernels. ${ }^{61}$ The output response up to second order is rewritten as

$$
\begin{aligned}
y(t)= & \sum_{j=1}^{m} \int_{-\infty}^{t} H_{1}^{x_{j}}(t-\tau) x_{j}(\tau) d \tau+ \\
& \sum_{j_{1}=1}^{m} \sum_{j_{2}=1}^{m} \int_{-\infty}^{t} \int_{-\infty}^{t} H_{2}^{x_{j_{1}}, x_{j_{2}}}\left(t-\tau_{1}, t-\tau_{2}\right) x_{j_{1}}\left(\tau_{1}\right) x_{j_{2}}\left(\tau_{2}\right) d \tau_{1} d \tau_{2}+\mathcal{O}\left(|x|^{3}\right)
\end{aligned}
$$

Note that the superscripts in Eq. (8) identify to which inputs the kernel corresponds, for example, the second-order kernel $H_{2}^{x_{j_{1}}, x_{j_{3}}}$ correlates the inputs $x_{j_{1}}$ and $x_{j_{3}}$. Note that the second and higher-order kernels are symmetric with respect to the arguments, $H_{2}^{x_{j_{1}}, x_{j_{3}}}=H_{2}^{x_{j_{3}}, x_{j_{1}}}$. The determination of Volterra kernels using CFD simulations is discussed in the section on System Identification.

\section{ROMs Based on Indicial Functions}

Linear and nonlinear models based on indicial functions are detailed here. Assuming a linear relationship between the input function and the output, a linear ROM is defined as the convolution (or Duhamel's superposition $^{30}$ ) of the indicial response with the derivative of the forcing function ${ }^{62}$

$$
y(t)=\frac{d}{d t}\left[\int_{0}^{t} A(t-\tau) x(\tau) d \tau\right]
$$

where $A$ represents the unit response, or indicial response, of the system. Note that a linear Volterra system and the linear step-type ROM given in Eq. (9) are identical. For a linear system, $H_{1}$ is the impulse response function and $H_{1}(t)=d A(t) / d t$ applies. The indicial response functions are used as a fundamental approach to represent the unsteady aerodynamic loads. The mathematical models are detailed by Tobak et al. ${ }^{14,15}$ and Reisenthel et al. ${ }^{23,24}$ The pitch moment is only considered in this paper as it is the more difficult parameter to predict. If the time responses in aerodynamic coefficients due to the step changes in angle of attack, $\alpha$, and angular velocity, $q$, are known, then the total produced pitch moment at time $t$ can be obtained using Eq. (10):

$$
C_{m}(t)=C_{m 0}+\frac{d}{d t}\left[\int_{0}^{t} C_{m \alpha}(t-\tau) \alpha(\tau) d \tau\right]+\frac{d}{d t}\left[\int_{0}^{t} C_{m q}(t-\tau) q(\tau) d \tau\right]
$$

where $C_{m 0}$ denotes the zero angle of attack pitch moment coefficient and is found from static calculations. For non-linear aerodynamic responses due to motions starting from different Mach numbers, the dependencies on the angle of attack and Mach number are added to the indicial functions, i.e. :

$$
C_{m}(t)=C_{m 0}(M)+\frac{d}{d t}\left[\int_{0}^{t} C_{m \alpha}(t-\tau, \alpha, M) \alpha(\tau) d \tau\right]+\frac{d}{d t}\left[\int_{0}^{t} C_{m q}(t-\tau, \alpha, M) q(\tau) d \tau\right]
$$


where $M$ denotes the free-stream Mach number. The response function due to pitch rate, i.e. $C_{m q}(\alpha, M)$ can be estimated using a time-dependent interpolation scheme from the observed responses. This value is next used to estimate the second integral in Eq.11] however, the estimation of the integral with respect to the angle of attack needs more explanation. Assuming a set of angle of attack samples of $\alpha=\left[\alpha_{1}, \alpha_{2}, \ldots, \alpha_{n}\right]$ at freestream Mach numbers of $M=\left[M_{1}, M_{2}, \ldots, M_{m}\right]$, the pitch moment response to each angle of $\alpha_{i}, i=1,2, \ldots, n$ at Mach number of $M_{j}, j=1,2, \ldots, m$ is denoted as $A_{\alpha}\left(t, \alpha_{i}, M_{j}\right)$. In these response simulations, $\alpha(t)=0$ at $t=0$ and is held constant at $\alpha_{i}$ for all $t>0$. For a new angle of $\alpha^{*}>0$ at a new free-stream Mach number of $M^{*}$, the responses of $A_{\alpha}\left(t, \alpha_{k}, M^{*}\right)$ are being interpolated at $\alpha_{k}=\left[\alpha_{1}, \alpha_{2}, \ldots, \alpha_{s}\right]$, such that $0<\alpha_{1}<\alpha_{2}<\ldots<\alpha_{s}$ and $\alpha_{s}=\alpha^{*}$. These angles can have a uniform or non-uniform spacing. The indicial functions of $C_{m \alpha_{k}}$ for $k=1, \ldots, s$ at each interval of $\left[\alpha_{k-1}, \alpha_{k}\right]$ are defined as

$$
\begin{aligned}
C_{m \alpha_{1}} & =\frac{A_{\alpha}\left(t, \alpha_{1}, M^{*}\right)-C_{m 0}}{\alpha_{1}} \\
C_{m \alpha_{k}} & =\frac{A_{\alpha}\left(t, \alpha_{k}, M^{*}\right)-A_{\alpha}\left(t, \alpha_{k-1}, M^{*}\right)}{\alpha_{k}-\alpha_{k-1}}
\end{aligned}
$$

where $C_{m 0}$ denotes the zero angle of attack pitch moment coefficient. The interval indicial functions are then used to estimate the values of first integral in Eq. 11] These steps can easily be followed for a negative angle of attack, i.e. $\alpha^{*}<0$. Once this model is created, the aerodynamic response to a wide range of motions can be predicted on the order of few seconds, however, the above model still requires a special time-dependent surrogate model to predict response functions at each $\alpha^{*}$ and $M^{*}$ from some available samples.

\section{Surrogate-Based Modeling of Indicial Functions}

Having a ROM to predict the aerodynamic responses to any arbitrary motion over a wide flight regime could become a very expensive approach because a large number of indicial functions needs to be computed. In order to achieve a reasonable computational cost, a special time-dependent surrogate-based modeling approach is adapted to predict indicial responses for a new point from available (observed) responses. These observed responses are viewed as a set of time-correlated spatial processes where the output is considered a time-dependent function. Romero et al. ${ }^{63}$ developed a framework for multi-stage Bayesian surrogate models for the design of time dependent systems and tested their model for free vibrations of a mass-spring-damper system assuming the input parameters of stiffness and damping factor at different initial conditions. This framework is examined for reduced order modeling of nonlinear and unsteady aerodynamic loads. Assume an input vector of $\mathbf{x}(t)=\left(x_{1}(t), x_{2}(t), \ldots, x_{n}(t)\right)$ where $n$ represents the dimensionality of the input vector. To construct a surrogate model for fitting the input-output relationship, the unsteady aerodynamic responses corresponding to a limited number of input parameters (training parameters or samples) need to be generated. Design of Experiment methods, for example, can be used to select $m$ samples from the input space. The input matrix $\mathbf{D}(m \times n)$ is then defined as:

$$
\mathbf{D}=\left[\begin{array}{cccc}
x_{11} & x_{12} & \cdots & x_{1 n} \\
x_{21} & x_{22} & \cdots & x_{2 n} \\
\vdots & \vdots & \vdots & \vdots \\
x_{m 1} & x_{m 2} & \cdots & x_{m n}
\end{array}\right]
$$

where rows correspond to different combinations of the design parameters. For each row in the input matrix, a time-dependent response was calculated at $p$ discrete values of time, and this information is summarized in the output matrix of $\mathbf{Z}(m \times p)$ as:

$$
\mathbf{Z}=\left[\begin{array}{cccc}
y_{11} & y_{12} & \cdots & y_{1 p} \\
y_{21} & y_{22} & \cdots & y_{2 p} \\
\vdots & \vdots & \vdots & \vdots \\
y_{m 1} & y_{m 2} & \cdots & y_{m p}
\end{array}\right]
$$


where for aerodynamic loads modeling, $p$ equals the number of iterations used in time-marching CFD calculations. The objective of surrogate modeling is to develop a model that allows predicting the aerodynamic response of $\mathbf{y}\left(\mathbf{x}_{\mathbf{0}}\right)=\left(y_{01}, y_{02}, \ldots, y_{0 p}\right)$ at a new combination of input parameter of $\mathbf{x}_{\mathbf{0}}$. To construct this surrogate model, the responses at each time step are assumed as a separate set, such that each column of the output matrix is a partial realization of the total response. In this sense, $p$ surrogate models are created; they are denoted as $\mathbf{Z}_{\mathbf{i}}(\mathbf{D})$ for $i=1,2, \ldots, p$. A universal-type Kriging function ${ }^{34}$ is used to approximate these models. Each $\mathbf{Z}_{\mathbf{i}}(\mathbf{D})$ function can be approximated as the sum of a deterministic mean (trend), $\mu$ and a zero-mean spatial random process of $\epsilon$ with a given covariance structure of $\sigma^{2}$, therefore each function value at the new sample of $\mathbf{x}_{\mathbf{0}}$ is

$$
\tilde{\mathbf{Z}}_{\mathbf{i}}\left(\mathbf{x}_{\mathbf{0}}\right)=\mu+\epsilon
$$

where, the tilde accent shows that surrogate model is an approximation of the actual function. Universal Kriging, which is used in this paper, assumes that the mean value $\mu$ is a linear combination of known regression functions of $\mathbf{f}_{0}(x), \mathbf{f}_{1}(x), . ., \mathbf{f}_{n}(x)$. In this paper, the linear functions are used, therefore, $\mathbf{f}_{0}(x)=1$ and $\mathbf{f}_{j}(x)=x_{j}$ for $j=1,2, \ldots, n$. This changes Eq. (16) as:

$$
\tilde{\mathbf{Z}}_{\mathbf{i}}\left(\mathbf{x}_{\mathbf{0}}\right)=\sum_{j=0}^{n} \beta_{i j} \mathbf{f}_{j}\left(\mathbf{x}_{\mathbf{0}}\right)+\epsilon
$$

where $\beta_{i j}$ represent the regression coefficient for the $j$-th regression function of response function at time step $i, i=1,2, \ldots, p$. To estimate the spatial random process of $\epsilon$, a spatially weighted distance formula is defined between samples given in matrix $\mathbf{D}$ such that for sample $\mathbf{x}_{i}$ and $\mathbf{x}_{j}$, the distance is written as:

$$
d\left(\mathbf{x}_{i}, \mathbf{x}_{j}\right)=\sum_{h=1}^{n} \theta_{h}\left|x_{h}^{(i)}-x_{h}^{(j)}\right|^{p_{h}} \quad\left(\theta_{h} \geq 0 \text { and } p_{h} \in[0,1]\right)
$$

where |.| shows the Euclidean distance; the parameter $\theta_{h}$ expresses the importance of the $h$-th component of the input vector, and the exponent $p_{h}$ is related to the smoothness of the function in coordinate direction $h$. A correlation matrix $\mathbf{R}(m \times m)$ with a Gaussian spatial random process is then defined as:

$$
\mathbf{R}=\left[\begin{array}{cccc}
\exp \left[-\frac{d\left(\mathbf{x}_{1}, \mathbf{x}_{1}\right)}{\sigma^{2}}\right] & \exp \left[-\frac{d\left(\mathbf{x}_{1}, \mathbf{x}_{2}\right)}{\sigma^{2}}\right] & \cdots & \exp \left[-\frac{d\left(\mathbf{x}_{1}, \mathbf{x}_{m}\right)}{\sigma^{2}}\right] \\
\vdots & \vdots & \vdots & \vdots \\
\exp \left[-\frac{d\left(\mathbf{x}_{m}, \mathbf{x}_{1}\right)}{\sigma^{2}}\right] & \exp \left[-\frac{d\left(\mathbf{x}_{m}, \mathbf{x}_{2}\right)}{\sigma^{2}}\right] & \cdots & \exp \left[-\frac{d\left(\mathbf{x}_{m}, \mathbf{x}_{m}\right)}{\sigma^{2}}\right]
\end{array}\right]
$$

To compute the Kriging model, values must be estimated for $\beta_{i j}, \sigma, \theta_{h}$, and $p_{h}$. These parameters can be quantified using the maximum likelihood estimator, as described by Jones et al. ${ }^{64}$ Next the vector of $\mathbf{R}(m \times 1)$ is defined from correlations between the new design parameter $\mathbf{x}_{\mathbf{0}}$ and the $m$ sample points, based on the distance formula in Eq. (18), i.e.

$$
\mathbf{r}=\left[\begin{array}{c}
\exp \left[-\frac{d\left(\mathbf{x}_{1}, \mathbf{x}_{0}\right)}{\sigma^{2}}\right] \\
\exp \left[-\frac{d\left(\mathbf{x}_{2}, \mathbf{x}_{0}\right)}{\sigma^{2}}\right] \\
\vdots \\
\exp \left[-\frac{d\left(\mathbf{x}_{m}, \mathbf{x}_{0}\right)}{\sigma^{2}}\right]
\end{array}\right]
$$

and now $\tilde{\mathbf{Z}}_{\mathbf{i}}\left(\mathbf{x}_{\mathbf{0}}\right)$ can be estimated as

$$
\tilde{\mathbf{Z}}_{\mathbf{i}}\left(\mathbf{x}_{\mathbf{0}}\right)=\sum_{j=0}^{n} \beta_{i j} \mathbf{f}_{j}\left(\mathbf{x}_{\mathbf{0}}\right)+\mathbf{r}^{T} \mathbf{R}^{-1}\left(\mathbf{Z}_{\mathbf{i}}(\mathbf{D})-\mathbf{F} \beta\right)
$$


where, $\beta$ is the $n+1$ dimensional vector of regression coefficients; $\mathbf{Z}_{\mathbf{i}}(D)$ is the observed responses at time step $i, i=1,2, \ldots, p$ and matrix $\mathbf{F}$ is

$$
\mathbf{F}=\left[\begin{array}{cccc}
\mathbf{f}_{\mathbf{0}}\left(x_{1}\right) & \mathbf{f}_{\mathbf{1}}\left(x_{1}\right) & \cdots & \mathbf{f}_{\mathbf{n}}\left(x_{1}\right) \\
\vdots & \vdots & \vdots & \vdots \\
\mathbf{f}_{\mathbf{0}}\left(x_{m}\right) & \mathbf{f}_{\mathbf{1}}\left(x_{m}\right) & \cdots & \mathbf{f}_{\mathbf{n}}\left(x_{m}\right)
\end{array}\right]
$$

The total response at $\mathbf{x}_{\mathbf{0}}$ is then combination of predicted values of each surrogate model, i.e.

$$
\tilde{\mathbf{Z}}\left(\mathbf{x}_{\mathbf{0}}\right)=\left(\tilde{\mathbf{Z}}_{\mathbf{1}}\left(\mathbf{x}_{\mathbf{0}}\right), \tilde{\mathbf{Z}}_{\mathbf{2}}\left(\mathbf{x}_{\mathbf{0}}\right), \ldots, \tilde{\mathbf{Z}}_{\mathbf{p}}\left(\mathbf{x}_{\mathbf{0}}\right)\right)
$$

\section{E. ROMs Based on Surrogate-Based Recurrence-Framework}

The set of non-linear equations describing the CFD system can be interpreted as a general representation of a non-linear, time-invariant, and discrete-time dynamical system. The state vector consists of the conservative variables, and its size is proportional to the number of grid points. In this study, the aerodynamic loads form the vector of outputs, which are not only a function of the instantaneous values of the inputs, but also a function of the time history of the inputs.

To generate a computationally efficient approximation of the unsteady aerodynamic loads without solving the expensive CFD equations, the form of a dynamical system is assumed. ${ }^{50}$ When the state vector of the full-order system is finite in dimension, the following non-linear system is equivalent to the unsteady CFD equations

$$
y(t)=\Phi(\mathbf{x}(t), \mathbf{x}(t-\Delta t), \ldots, \mathbf{x}(t-m \Delta t), y(t-\Delta t), \ldots, y(t-n \Delta t))
$$

where $\mathbf{x}$ takes the form of Eq. (6), and the function $\Phi$ maps the inputs to the outputs. The terms $m$ and $n$ represent the number of previous values of the external inputs and outputs, respectively, influencing the output at the current time instant. These parameters account for time-history effects and phase-lag in the flow development.

Central to the generation of the reduced-order model is the computation of the function $\Phi$. Without a closed-form analytical expression, a numerical approximation of $\Phi$ is constructed using a number of CFD solutions. For the pitching aircraft, any motion can be expressed as function of three parameters, e.g., $\alpha_{0}, \alpha_{A}$, and $k$ that represent mean angle of attack, amplitude, and reduced frequency, respectively. These independent variables form a parameter space, which represents the envelope of all possible flow conditions in which the aircraft configuration is expected to operate. To generate a consistent set of unsteady aerodynamic loads in response to a given aircraft motion time history, the training cases at which CFD solutions are calculated should be representative of the expected flow conditions. Several Design of Experiment methods are available in the literature. A description of the Kriging-based framework used in this study is detailed in Ref. ${ }^{65}$ Let $N_{T}$ be the number of training cases at which CFD solutions are available. Each training case consists of different combinations of the independent parameters,

$$
\mathbf{x}_{i}=\left(\alpha_{i}(t), \dot{\alpha}_{i}(t), \ddot{\alpha}_{i}(t), \ldots\right) \quad \text { for } i=1, \ldots, N_{T}
$$

and the corresponding aerodynamic loads are indicated by $y_{i}(t)$. The approximation of the function $\Phi$ is obtained by interpolating the sampled data in the form of an input/output relationship. Several interpolation methods are available in the literature, and two of these have been used in the present study. Kriging interpolation is a common choice, but for increasing number of independent parameters the problem can result to be ill-conditioned. An alternative approach is the multi-linear interpolation technique, which is in general faster than the Kriging interpolation.

\section{F. ROMs based on RBFNN}

The nonlinear and unsteady aerodynamics can be viewed as a multi-input/multi-output dynamic system with a reconstructed state space model given by Eq. (24). The function $\Phi$ is approximated through RBF 
neural networks from a set of training data. RBF network provides an approximation of the functions based on the location of data points, and is generally much faster than multi-layer feed-forward neural networks. ${ }^{66}$ Given an input vector of $\left\{X_{j}^{c}: j=1, \ldots, p\right\}, X_{j}^{c} \in \mathbf{R}$ and a corresponding output vector of $\left\{Y_{j}^{c}: j=1, \ldots, p\right\}, Y_{j}^{c} \in \mathbf{R}$, the RBF approximates the output at a new given point as:

$$
\hat{Y}\left(X^{*}\right)=\sum_{\mathrm{k}=1}^{P} \alpha_{\mathrm{k}} \Phi_{i}(X)
$$

such that

$$
\hat{Y}\left(X_{j}^{c}\right)=Y_{j}^{c}, \text { for } \mathrm{j}=1,2, \ldots, p
$$

where $\alpha_{\mathrm{k}}$ are the weights of the linear combiners. The functions $\Phi_{i}$ are named Radial Basis Functions and are often described by a Gaussian basis function as:

$$
\Phi_{i}(X)=\exp \left(-\frac{\left\|X^{*}-X_{j}^{c}\right\|^{2}}{\beta^{2}}\right)
$$

where, $\beta$ is a real variable to be chosen by the user, and $\|$. $\|$ denotes the Euclidean norm such that the functions $\Phi_{i}$ will vanish at sufficiently large values of $\left\|X^{*}-X_{j}^{c}\right\|$. In terms of the network structure, the RBFNN is a two-layer processing structure with one hidden layer that approximates $\Phi_{i}$ at each node. Then, the output layer is a set of linear combiners of approximation from hidden layer nodes. The network is then trained to minimize the error between the target (desired) values and the network predicted values.

\section{G. System Identification}

The identification of the Volterra kernels is performed using an unsteady time-domain simulation as the source of the data. The CFD solution is discrete in time, and the time-step is indicated by $\Delta t^{*}$. Denote the input vector at each time step as $x[n]=x\left(n \Delta t^{*}\right)=x(t)$. The discrete-time representation of Eq. (8) is

$$
\begin{aligned}
y[n]= & \sum_{j=1}^{m} \cdot \sum_{k=0}^{n} H_{1}^{x_{j}}[n-k] x_{j}[k]+ \\
& \sum_{j_{1}=1}^{m} \sum_{j_{2}=1}^{m} \cdot \sum_{k_{1}=0}^{n} \sum_{k_{2}=0}^{n} H_{2}^{x_{j_{1}}, x_{j_{2}}}\left[n-k_{1}, n-k_{2}\right] x_{j_{1}}\left[k_{1}\right] x_{j_{2}}\left[k_{2}\right]+\mathcal{O}\left(|x|^{3}\right)
\end{aligned}
$$

The computational determination of system impulse response may be complicated as it occurs over a very short period of time, ${ }^{67}$ thus, it is more common to use indirect methods. The indirect estimation of Volterra kernels involves the resolution of an overdetermined system. Values of aerodynamic coefficients and the time-history of the motion variables are known from the CFD simulation used as training input. Let $\mathbf{y}=(y[0], y[1], \ldots, y[n])^{T}$ denote each aerodynamic load computed using CFD, and let $\mathbf{A}$ contain the permutations of input parameters relevant to the unsteady motion. Equation (29) can be recast in the form

$$
\mathbf{y}=\mathbf{A} \mathbf{b}
$$

where the vector $\mathbf{b}$ contains the unknown Volterra kernels. The matrix $\mathbf{A}$ is in general non-square, with more rows than columns. Several numerical methods are available to solve least squares problems, e.g., direct inversion of $\mathbf{A}^{T} \mathbf{A}$, Gauss elimination, Moore-Penrose generalized inverse approach and the QR factorization. However, the Moore-Penrose approach and the QR factorization are more accurate than the Gaussian elimination and the direct inversion solutions. The cost of the QR factorization is $\mathcal{O}\left(n^{2}\right)$, and the Moore-Penrose inversion involves $\mathcal{O}\left(n^{3}\right)$ operations. Note that computational resources attributable to the identification of the Volterra kernels grow exponentially with order. Increasing the order of the Volterra series introduces a requirement for a training manoeuvre of sufficient duration. A remedy to this is the use of a simplified form of the kernel parametric structure. For example, Maciej et al ${ }^{68}$ proposed to set all off-diagonal terms of the kernel to zero, i.e. 


$$
\begin{aligned}
& H_{p}^{x_{j_{1}}, x_{j_{2}}, \ldots, x_{j_{p}}}\left[n-k_{1}, n-k_{2}, \ldots, n-k_{p}\right]=0 \\
& \quad \text { for } k_{1} \neq k_{2} \neq \ldots \neq k_{p}
\end{aligned}
$$

In this work, the Volterra kernels are identified from Eq. (30) solving for $\mathbf{b}$, with $\mathbf{y}$ and $\mathbf{A}$ being known for a training maneuver. The matrix $\mathbf{A}$ is then recomputed for a novel maneuver, and the low-order model in Eq. (30) is used to predict the resulting unsteady aerodynamic loads in place of the full-order system.

An approach based on grid motion is used to calculate the indicial response directly from CFD. This approach allows the uncoupling of effects of angle of attack and pitch rate for the indicial functions. Cobalt uses an arbitrary Lagrangian-Eulerian formulation and hence allows all translational and rotational degrees of freedom. ${ }^{32}$ The code can simulate both free and specified six degree of freedom $(6 \mathrm{DoF})$ motions. The rigid motion is specified from a motion input file. For the rigid motion the location of a reference point on the aircraft is specified at each time step. In addition the rotation of the aircraft about this reference point is also defined using the rotation angles of yaw, pitch and bank. The aircraft reference point velocity, $v_{a}$, in an inertial frame is calculated to achieve the required angles of attack and sideslip, and the forward speed. The velocity is then used to calculate the location. The initial aircraft velocity, $v_{0}$, is specified in terms of Mach number, angle of attack and side-slip angle in the main file. The instantaneous aircraft location for the motion file is then defined from the relative velocity vector, $v_{a}-v_{0}$. For CFD-type calculation of a step change in angle of attack, the grid immediately starts to move at $t=0$ to the right and downward. The translation continues over time with a constant velocity vector. Since there is no rotation, all the effects in aerodynamic loads are from changes in the angle of attack. For a unit step change in pitch rate, the grid moves and rotates simultaneously. The grid starts to rotate with a unit pitch rate at $t=0$. To hold the angle of attack zero during the rotation, the grid moves right and upward.

A training maneuver(s) is needed to provide enough information to learn the mapping between input and output of ROMs given by Eq. (24). Previous studies to generate training maneuvers for aerodynamic characteristics $^{69-73}$ are limited by the range of the motion frequency content. A ROM identified from such a maneuver has limitations with respect to $\mathrm{S} \& \mathrm{C}$ applications. Thus, the basic requirement for a training maneuver to generate a reliable ROM in S\&C applications is that it sufficiently covers the desired regressor space of state variables. A ROM built on data produced by such motions can then be used to predict the aircraft aerodynamic behavior within the regressor space. The systematic coverage of the regressor space can be, in general, treated as an optimization problem of filling the multidimensional space with strong constraints resulting from the fact that some axes of the regressor space do not represent an independent variable. For the current study, special training maneuvers are considered: a linear chirp, spiral, and Schroeder. These maneuvers are defined in Table 1

Table 1. Special Training Maneuvers

\begin{tabular}{ll}
\hline \hline Maneuver & Definition \\
\hline Linear chirp & $\alpha(t)=\alpha_{0}+\alpha_{A} \sin \left(\omega t^{2}\right)$ \\
Spiral & $\alpha(t)=\alpha_{0}+\alpha_{A} t \sin (\omega t)$ \\
Schroeder & $\alpha(t)=\alpha_{0}+\alpha_{A} \sum_{k=1}^{n} \sqrt{\frac{1}{2 N}} \cos \left(\frac{2 \pi k t}{T}-\frac{\pi k^{2}}{N}\right)$ \\
\hline \hline
\end{tabular}

where $\alpha_{0}$ is the mean angle of attack; $\alpha_{A}$ is amplitude and $\omega$ is angular velocity. The chip maneuver used has a constant amplitude and linearly increasing frequency in time. In the spiral maneuver the amplitude increases linearly in time, so the angle of attack. The Schroeder maneuver is a multi-stage frequency sweep consisting of multiple cosine terms with a specified phasing. This maneuver has three parameters that enable direct control of the regressor space coverage. These are maneuver amplitude, $\alpha_{A}$, the maneuver length, $T$, and the number of frequencies in the maneuver, $N$.

\section{Test Case}

The X-31 aircraft is considered in this paper. The aircraft geometry and wind tunnel data were provided to the participants in NATO RTO Task Group AVT-161 (Assessment of Stability and Control prediction Methods for NATO Air and Sea Vehicles). ${ }^{74}$ The objective of this task group is to evaluate CFD codes 
against the wind tunnel results. The vehicle is a super-maneuverable fighter which was built by the United States and West Germany in the 1990s. The test aircraft has been a subject of extensive flight, and wind tunnel tests (see for example Canter and Groves, ${ }^{75}$ Alcorn et al., ${ }^{76}$ Williams et al. ${ }^{77}$ and Rein ${ }^{78}$ ) and CFD simulations (example is the work of Schütte et al. ${ }^{5}$ ). A three-view drawing of the vehicle is shown in Fig. 11. The aircraft has a fuselage length of $13.21 \mathrm{~m}$, a canard, and a double delta wing with total wing span of 7.26 $\mathrm{m}$. The inner delta wing has a sweep angle of $57^{\circ}$ and the outer sweep is $45^{\circ}$. The inner wing sweep places the wing behind the supersonic shock wave, while the outer one improves the vehicle stability and control. ${ }^{79}$ The canard is a cropped delta wing with a sweep angle of $45^{\circ}$. Additional characteristics of the model are the inner and outer leading edge flaps, the trailing edge flaps, the front wing and rear fuselage strakes.

The computational mesh was generated in two steps. In the first step, the inviscid tetrahedral mesh was generated using the ICEMCFD code. This mesh was then used as a background mesh by TRITET ${ }^{80,81}$ which builds prism layers using a frontal technique. TRITET rebuilds the inviscid mesh while respecting the size of the original inviscid mesh from ICEMCFD. The mesh overview is shown in Fig. 2 The grid is a symmetric configuration and contains 4.9 million points and 11.7 million cells. Three boundary conditions were imposed to the surfaces: a far-field, symmetry, and solid wall. The low-speed experiments are available from the DLR, German Aerospace Center. ${ }^{78}$ The wind tunnel model has a closed inlet and fitted with moving lift and control surfaces. The experiments are composed of two setups. The first setup uses a belly-mounted sting attached to the model directly under the main wing. This setup allows six degree of freedom motions. The second setup uses an aft mounted sting connected to an arm in the wind tunnel. The values of lift, drag, and pitch moment of second setup are used to validate CFD predictions. CFD simulations were run on the Cray XE6 (open system) machine at the Engineering Research Development Center (ERDC) [Machine name is Chugach with $2.3 \mathrm{GHz}$ core speed and 11,000 cores]. Four turbulence models were tested: the Spalart-Allmars (SA), ${ }^{82}$ the SA with Rotation Correction (SARC), ${ }^{83}$ the Menter's Shear Stress Transport $(\mathrm{SST})^{84}$ and SARC with Detached Eddy Simulations (SARC-DES). Figure 3 compares the lift, drag, and pitch moment coefficients obtained from each turbulence model with the available measurements. All these models yield similar predictions at low angles of attack, but they result in a wide spread of predictions at moderate to high angles. For angles between, $\alpha=15^{\circ}-23^{\circ}$, SARC-DDES and SST models perform quite well compared to SA and SARC. DES and SST accurately predict unsteady separated flows occurring at these angles, but for angles above $23^{\circ}$, all models fail to predict accurately the massively separated flows.

Some features of aerodynamic characteristics from the SARC-DES turbulence model predictions are explained. There is an emanating vortex from the canard tip at small angles of attack. This vortex is the source of the small non-linearity in the pitch moment at low angles of attack. As angle of attack is increased, the canard vortex becomes stronger, resulting in a negative pressure on the upper surface and forward movement of the aerodynamic center. Therefore, the pitch moment slope suddenly increases from the slope value at zero degrees angle of attack. This vortex is shown in Fig. 4(a) for 10 degrees angle of attack. Around an angle of 14 degrees, the canard vortex starts to breakdown and the wing vortex is formed as shown in Fig. 4(b). The wing vortex helps to further forward movement of aerodynamic center and increase of pitch moment. At 18 degrees angle of attack, the canard vortex breakdown point is nearly moved to the leading edge and then the wing vortex starts to breakdown as shown in Fig. 4(c). This results in an aftwards movement of aerodynamic center and a change in the pitch moment slope sign. The wing vortex breakdown point moves towards the leading edge by increasing angle of attack (Fig. 4(d)- (e)). The canard vortex is fully separated at these angles. As the vortex breakdown point becomes close to the wing leading edge (Fig. 4l(f)), the pitch moment starts to rise again. The SARC-DES turbulence model was used for all ROM computations.

\section{Results and Discussion}

All ROMs are first evaluated to predict the unsteady pitch moment resulting from a sinusoidal pitch oscillation at free-stream Mach number of 0.9 and reduced frequency of $k=0.01$. The amplitude of oscillation is held constant at seven degrees and the mean angle of attack is zero degrees. Figure [5(a) shows the computed pitch moment coefficient, $C_{m}$, by solving the RANS and SARC-DES turbulence model equations in a timeaccurate fashion. This solution and other time-accurate CFD simulations are labeled as "Time-Marching" in the ROM plots. The cost of simulating three pitch cycles is approximatly 52 wall-clock hours using 256 processors $(2.3 \mathrm{GHz})$. Figure 5(a) shows that the pitch moment curve makes a nonlinear loop on the figure of moment versus angle of attack due to occurrence of shock waves and vortices. This figure also shows that 
the pitch moment curve is not symmetric about zero degrees angle of attack. The moment curve shows a negative slope during the pitch cycle such that it has more negative slope values at negative angles of attack compared with the slope values at positive angles of attack. Some flow features during the pitch oscillation are shown in Figs. 5(b)-(g) and briefly discussed here. In Fig. 5(b) the angle of attack is $-2.1^{\circ}$ and a vortex can be seen emanating from the wing root on the lower surface which spirals towards the wing tip. This vortex causes a sharp negative pressure peak to occur close to the wing leading edge as shown in the surface pressure plots of Figs. [5 (b)-(d). Figure [5) also shows that a shock wave is formed on the lower surface of the wing which is nearly perpendicular to the fuselage before it interacts with the leading edge vortex. At the minimum angle of attack in the pitch cycle, i.e. $\alpha=-7^{\circ}$, the leading edge vortex becomes much stronger and the wing surface pressure close to the leading edge drops further as shown in Fig. 5)(c). This figure also shows that as the angle of attack becomes smaller, the shock moves downstream and therefore changes the pitch moment curve slope. No vortices were observed on the wing during pitching at positive angles of attack, but a vortex was formed on the canard tip at the maximum angle of attack in the pitch cycle, i.e. $\alpha=7^{\circ}$, as shown in Fig. 5(f). Figures (5) (e)-(g) show that a shock wave is formed over the upper surface which is no longer perpendicular to the fuselage and moves slowly with increasing in the angle of attack during upstroke.

For the identification of the Volterra kernels, the chirp and spiral training maneuvers were generated using CFD as the source of the data. The variation of angle of attack with time for these maneuvers is shown in Figs. 6] a) and (c). Both maneuvers ran for 2.4 seconds of physical time and started from a steady-state solution. The chirp maneuver has an amplitude of seven degrees starting from zero degrees angle of attack and pitching with a frequency of $1 \mathrm{~Hz}$ at $t=0$. Note that the chirp motion frequency increases linearly with time. The spiral maneuver has an initial amplitude of $3.5^{\circ}$, starting from zero degrees angle of attack and pitching at constant frequency of $k=0.01$. The oscillation amplitude in the spiral motion increases as time progresses. Note that the spiral maneuver is at the reduced frequency of the maneuver to be predicted. The cost of generating each training maneuver is approximatly 84 wall-clock hours using 256 processors. The first and second order kernels of the Volterra model were estimated from time-history simulations of chirp and spiral training maneuvers. These estimations were used next to predict the unsteady pitch moment data shown in Fig. 5(a). The ROM predictions based on spiral and chirp training maneuvers are compared with the time-accurate solution data in Figs. 6(b) and (d). The comparisons show a good agreement with CFD data for a ROM identified from spiral data, but the ROM identified from chirp data does not match well, in particular, around the maximum and minimum angles of attack. The instantaneous frequency in the chirp maneuver varies with time and hence it might not have sufficient information to identify the Volterra kernels corresponding to a swept amplitude motion at constant frequency. However, the ROM based on chirp data could be used for predicting aerodynamics responses from pitch oscillations at many other frequencies (those covered by the simulation of chirp training maneuver), but the ROM based on spiral is possibly valid for the motions at a fixed reduced frequency.

The generated chirp and spiral training maneuvers were also used to find a mapping between the pitch moment coefficient and the instantaneous pitch motion variables. This mapping was next learned using a RBF neural network. Also, a Schroeder maneuver was defined by a multi-stage frequency sweep. This maneuver started from an initial angle of attack of $4.95^{\circ}$. The number of frequencies in the maneuver, $N$, was set to 20 with an initial amplitude of seven degrees. This maneuver ran for 2.4 seconds of physical time as well and is shown in Fig. 7(e). The aircraft responses to these three maneuvers were generated using URANS equations. The training data were next normalized using the mean and standard deviation of each input. The data are then rearranged according to Eq. (24) and the RBF network performance is tested for different values of $m$ and $n$, with a performance error threshold of $1 \times 10^{-6}$. All networks computed converged to the threshold error. The results showed that using $m=n=4$ is sufficient for modeling the studied motions. The trained networks were then tested against the target motion; the ROM predictions are shown in Figs. 7(b),(d) and (f). These figures show that the predicted ROM values agree well with the time-marching solution, although the ROM based on Schroeder maneuver showed better accuracy than models based on the chirp and spiral maneuvers.

The indical pitch moment responses of the X-31 aircraft with a unit step change in angle of attack and pitch rate are shown in Fig. 8(a). These functions correspond to the fixed Mach number of 0.9. In $C_{L \alpha}$ simulations, the angle of attack is zero degrees at $t=0$ and is held constant to one degree for all other times. In $C_{L q}$ simulations, the grid starts to pitch up with a normalized pitch rate of $q=1 \mathrm{rad}$ at $t=0$ and the angle of attack is held to zero degrees during simulations with the aid of grid translation. All 
computations started from a steady-state solution and then advanced in time using second-order accuracy with five Newton subiterations. As shown in Fig. 8(a), the pitch moment responses have a negative peak at $t=0$ followed by an increasing trend. As the steady flow around the vehicle is disturbed by the grid motion, a compression wave and an expansion wave are formed on the lower and upper surface of the vehicle that cause a sharp negative pitch moment peak in the responses. ${ }^{32}$ As the response time progresses, the waves begin to move away from the vehicle and the pitch moment responses start to increase and then asymptotically reach the steady-state values. The cost of generating each indicial function is around 1.5 wall-clock hours using 256 processors. A linear ROM was created using Eq. (10) and used for prediction of target maneuver. The results are compared with full-order model in Fig. 8 (b). The figure shows that linear ROM fails to accurately predict the pitch moment values at all angle of attack. The functions of $C_{L \alpha}$ vary largely with angle of attack at transonic speed range and thus a linear ROM cannot predict these effects. Next, the X-31 $C_{L \alpha}$ functions were simulated at different angles of attack and at free-stream Mach number of 0.9 and shown in Fig. 8(c). Note that the pitch moment slope is not symmetric with zero angle of attack and hence the simulations included both positive and negative angle of attack responses. The total cost of generating a nonlinear ROM is now increased to approximatly 21 wall-clock hours. Fig. 8)(c) shows that the responses at initial time are invariant with angle of attack, but the intermediate and final values change depending on the angle of attack. Fig. 8(c) shows that the pitch moment responses have more negative values than positive angles of attack due to vortex formation on the lower surface of the wing. A nonlinear ROM was created and then using a linear interpolation scheme, the prediction of target maneuver was evaluated. Figure 8(d) shows that the nonlinear ROM predictions agree very well with full-order simulation values. Note that such a nonlinear ROM could be used for computing the pitch moment responses from many other motions with different amplitudes and frequencies. For example, the ROM was used to predict two pitch oscillations with four and six degrees amplitude at $M=0.9$ and $k=0.01$. The predictions are compared with time-marching solutions in Figs. 9(a) and (b). Again a very good match was found. Also, the ROM predictions were evaluated for the chirp, spiral and Schroeder maneuvers used in RBF work. Figs. 9](c)-(e) show that even for this varying amplitude and frequency motions, the created ROM matches very well with CFD data.

For the generation of the SBRF model, time-accurate simulations were pre-computed for various combinations of pitch amplitude and Mach number at a fixed reduced frequency. The two-dimensional parameter space was filled using Design of Experiement methods and is shown in Fig. 10(a). Also, the pitch motion simulations of all samples are shown in Fig. 10(b). The SBRF model was used for the prediction of the pitch moment coefficient time history for sinusoidal forced motions about zero degrees angle of attack, amplitude of seven degrees and values of Mach number of $0.78,0.825$, and 0.88 . Model predictions are compared to time-accurate results in Fig. 11. Tests were performed to evaluate the dependency of the model predictions on the number of previous steps in the inputs (angle of attack time history, first and second time derivatives, and Mach number) and output (the prediction itself). No significant dependence was found for values of $m$ and $n$ up to 2 . This may be attributed to the small time step increments used in the time-accurate simulations. Although the method robustness could degrade for higher values of $m$ and $n$, it is considered relevant that the predictions are unaffected for a range of values. In this work, the Kriging interpolation was used to approximating the mapping function between inputs and outputs. A good agreement is noted in Fig. 11 for all flight conditions, with the model predictions being generated in few seconds.

An issue regarding SBRF model is that cost of simulating three pitch cycles for each sample shown in Fig. 10(a) is around 52 wall-clock hours and the model still cannot predict the aerodynamic responses to motions at other frequencies. A ROM based on indicial functions, along with a time-dependent surrogate approach, is proposed for aerodynamics modeling in the angle of attack/Mach number/frequency space. In this model, the indicial functions in the angle of attack and Mach number space are interpolated from some available samples. Note that the ROM based on these functions is still cheaper than the full-order model and SBRF model because the indicial functions eliminate the need of repeating calculation for each frequency. The samples could be generated using methods of factorial design, Latin hypercube sampling, low discrepency sequences, and designs based on statistical optimality criteria. ${ }^{85}$ Factorial designs are extremely simple to construct and have been used in this work. The X-31 motions considered encompass $\alpha$ and $M$ values in the range of $\left[-7^{\circ}, 7^{\circ}\right]$ and $[0.75,0.9]$, respectively. A set of samples including 56 points is defined on the $\alpha$ and $M$ space using factorial design. These points are shown in Fig. 12(a). The indicial functions with respect to angle of attack are calculated using the CFD and grid motion approach for each sample conditions. The pitch rate indicial functions are calculated for a unit step change in the pitch rate for each Mach number shown in 
Fig. 12(a). The total cost of generating all functions is now approximatly 90 hours. The calculated indicial functions due to a unit step change in angle of attack are shown in Fig. 12(b) for each Mach number in the sample design. This figure shows that the pitch moment initial, intermediate, and final loadings are different at each Mach number. The initial peak in the pitch moment becomes smaller for higher Mach number. An explanation is given by Leishman; ${ }^{86}$ this is due to the propagation of pressure disturbances at the speed of sound. Note that the pitch moment responses suddenly change for Mach numbers above 0.85 due to shock formation. Fig. 12(c) also shows that pitch rate indicial functions decrease as Mach number increases. A new ROM is now created along with a time-dependent surrogate model to determine the terms in Eq. [1] at each time step. The validity of ROM is tested for several motions in the angle of attack/frequency/Mach number space and compared with time-accurate CFD simulations in Fig. 13] This figure shows that the ROM predictions agree well with the CFD data. Small discrepancies are found in the high speed motions. This is likely due to the sample design used with a uniform spacing and the fact that pitch moment changes suddenly at high speeds. More samples at high speeds could improve the model predictions. The future work extends the results to include different sampling strategies. Finally, the created ROM could predict many motions in the angle of attack/frequency/Mach number space within a few seconds.

\section{Conclusions}

The aircraft stability and control analysis requires a very large number of CFD simulations to determine appropriate forcing parameters within the frequency/amplitude/Mach number space. Typically, the timeaccurate CFD simulations start from a steady state solution and are marched (iterated) in pseudo time within each physical time step using a dual-time stepping scheme. Also, to have a free decay response to the initial grid perturbation, it is often necessary to march time-accurate solution for several oscillations. Also, the configuration used in this work, the X-31 aircraft, has highly swept slender wings resulting in complex vortical flow under various conditions. A highly refined mesh, small time step, and the use of hybrid turbulence models such as Detached-Eddy Simulation and Delayed Detached-Eddy simulation are required to accurately resolve the unsteady flow around the aircraft in space and time. Because of the combination of large grids, small time steps, hybrid turbulence models, and a large number of simulations, the full-order modeling approach is too expensive for stability and control analysis of aircraft. This paper investigates the use of reduced order models that significantly reduce the CFD simulation time required to create a full aerodynamics database, making it possible to accurately model aircraft static and dynamic characteristics from a limited number of time-accurate CFD simulations.

The models considered were based on Duhamel's superposition integral using indicial (step) response functions, Volterra theory using nonlinear kernels, Radial Basis functions, and a surrogate-based recurrence framework, both using time-history simulations of a training maneuver(s). The indicial functions were directly calculated from unsteady RANS simulations starting from an initial steady-state condition with a prescribed grid motion. An important feature of this approach is uncoupling the effects of angle of attack and pitch rate into responses from pitching motions. A method to efficiently reduce the number of step function calculations within the angle of attack/Mach number space was described. This method uses a time-dependent surrogate model to fit the relationship between flight conditions (Mach number and angle of attack) and step functions calculated for a limited number of samples. An indirect method was proposed to estimate the nonlinear Volterra kernels from time-accurate computational fluid dynamic simulations of chirp and spiral training maneuvers. These maneuvering simulations were also used to estimate the unknown parameters in a model based on Radial Basis functions. A Design of Experiment method was used to generate several pitching motions at different amplitudes and free-stream Mach numbers. The model based on a surrogate-based recurrence framework then approximated the aerodynamic responses induced by pitching motions at new amplitides and Mach numbers. Overall, the reduced order models were found to produce accurate results, although a nonlinear model based on indicial functions yielded the best accuracy among all models. This model, along with a developed time-dependent surrogate approach, helped to produce accurate predictions for a wide range of motions in the transonic speed range. The cost of generating each motion using time-accurate CFD simulations was approximatly 52 wall-clock hours using 256 processors $(2.3 \mathrm{GHz})$ but the reduced order model predictions were within a few seconds. The future work extends the results to include different sampling strategies and will include aerodynamics modeling of lateral forces and moments as well. 


\section{Acknowledgements}

Mehdi Ghoreyshi was supported by the National Research Council/US Air Force Office of Scientific Research. Computer time was provided by the Department of Defence Engineering Research Development Center (ERDC). The USAFA authors appreciate the support provided by the Modeling and Simulation Research Center.

\section{References}

${ }^{1}$ Nelson, R. C. and Pelletier, A., "The Unsteady Aerodynamics of Slender Wings and Aircraft Undergoing Large Amplitude Maneuvers," Progress in Aerospace Sciences, Vol. 39, No. 2, 2003, pp. 185-284.

${ }^{2}$ Silva, W. A., "Application of Nonlinear Systems Theory to Transonic Unsteady Aerodynamics Responses," Journal of Aircraft, Vol. 30, No. 5, 1993, pp. 660-668.

${ }^{3}$ Tobak, M. and Schiff, L. B., "On the Formulation of the Aerodynamic Characteristics in Aircraft Dynamics," NASA TR $\mathrm{R}-456,1976$ 2007.

${ }^{4}$ Liebe, R., Flow Phenomena in Nature: A Challenge to Engineering Design, WIT Press, Southampton, Great Britain,

${ }^{5}$ Schütte, A., Einarsson, G., Raichle, A., Schoning, B., Mönnich, W., and Forkert, T., "Numerical Simulation of Manoeuvreing Aircraft by Aerodynamic, Flight Mechanics, and Structural Mechanics Coupling," Journal of Aircraft, Vol. 46, No. 1, 2009, pp. 53-64.

${ }^{6}$ Ghoreyshi, M., Vallespin, D., Da Ronch, A., Badcock, K. J., Vos, J., and Hitzel, S., "Simulation of Aircraft Manoeuvres based on Computational Fluid Dynamics," AIAA Paper 2010-8239, August 2010.

${ }^{7}$ Ghoreyshi, M., Jirásek, A., and Cummings, R. M., "CFD Modeling for Trajectory Predictions of a Generic Fighter Configuration," AIAA Paper 2011-6523, August 2011.

${ }^{8}$ Chin, S. and Lan, C. E., "Fourier Functional Analysis for Unsteady Aerodynamic Modeling," AIAA Journal, Vol. 30, No. 9, 1992, pp. 2259-2266.

${ }^{9}$ Lisandrin, P., Carpentieri, G., and van Tooren, M., "Investigation over CFD-based Models for the Identification of Nonlinear Unsteady Aerodyanmics Responses," AIAA Journal, Vol. 44, No. 9, 2006, pp. 2043-2050

${ }^{10}$ McDaniel, D. R., Cummings, R. M., Bergeron, K., Morton, S. A., and Dean, J. P., "Comparisons of CFD Solutions of Static and Maneuvering Fighter Aircraft with Flight Test Data," Journal of Aerospace Engineering, Vol. 223, No. 4, 2009, pp. 323-340.

${ }^{11}$ Lucia, D. J., Beran, P. S., and Silva, W. A., "Reduced-Order Modeling: New Approaches For Computational Physics," Progress in Aerospace Sciences, Vol. 40, 2004, pp. 51-117.

12 Jategaonkar, R. V., Flight Vehicle System Identification, 2006, AIAA Educational series, Vol. 216, Reston, VA.

${ }^{13}$ Ballhaus, W. F. and Goorjian, P. M., "Computation of Unsteady Transonic Flow by the Indicial Method," AIAA Journal, Vol. 16, No. 2, 1978, pp. 117-124.

${ }^{14}$ Tobak, M., Chapman, G. T., and Schiff, L. B., "Mathematical Modeling of the Aerodynamic Characteristics in Flight Dynamics," NASA TN-85880, 1984

${ }^{15}$ Tobak, M. and Chapman, G. T., "Nonlinear Problems in Flight Dynamics Involving Aerodynamic Bifurcations," NASA $\mathrm{TN}-86706,1985$

${ }^{16}$ Hall, K. C., Thomas, J. P., and Dowell, E. H., "Reduced-order Modelling of Unsteady Small-Disturbance Using a Frequency-Domain Proper Orthogonal Decomposition Technique," AIAA Paper 1999-655, Jan 1999.

${ }^{17}$ Gaitonde, A. and Jones, D. P., "Reduced Order State-Space Models from the Pulse Responses of a Linearized CFD Scheme," International Journal of Numerical Methods in Fluids, Vol. 42, No. 6, 2003, pp. 581-606.

${ }^{18}$ Silva, W. A. and Bartels, R. E., "Development of Reduced-Order Models for Aeroelastic Analysis and Flutter Prediction Using the CFL3Dv6.0 Code," Journal of Fluids and Structure, Vol. 19, No. 6, 2004, pp. 729-745.

${ }^{19}$ Jirásek, A., Jeans, T. L., Martenson, M., Cummings, R. M., and Bergeron, K., "Improved Methodologies For Maneuver Design of Aircraft Stability and Control Simulations," AIAA Paper 2010-515, Jan 2010.

${ }^{20}$ Goman, M. G. and Khrabov, A. N., "State-Space Representation of Aerodynamic Characteristics of An Aircraft at High Angles of Attack," AIAA Paper 92-4651, August 1992.

${ }^{21}$ Juang, J., "System Identification of a Vortex Lattice Aerodynamic Model," NASA TM-2001-211229, October 2001.

${ }^{22}$ Leishman, J. G. and Nguyen, K. Q., "State-Space Representation of Unsteady Airfoil Behavior," AIAA Journal, Vol. 28, No. 5, 1989, pp. 836-844.

${ }^{23}$ Reisenthel, P. H., "Development of a Nonlinear Indicial Model Using Response Functions Generated by a Neural Network," AIAA Paper 97-0337, Jan 1997.

${ }^{24}$ Reisenthel, P. H. and Bettencourt, M. T., "Data-based Aerodynamic Modeling Using Nonlinear Indicial Theory," AIAA Paper 97-0337, Jan 1999

${ }^{25}$ Ghoreyshi, M. and Cummings, R. M., "Aerodynamics Modeling of a Maneuvering Aircraft Using Indicial Functions," AIAA Paper 2012-689, Jan 2012. 2006.

${ }^{26}$ Librescu, L. and Song, O., Thin-Walled Composit Beams: Theory and Application, Springer, Dordrecht, Netherland,

${ }^{27}$ Manglano-Villamarine, C. E. and Shaw, S. T., "Three-dimensional Indicial Response of Finite Aspect Ratio Yawed Wings," The Aeronautical Journal, Vol. 111, No. 1120, 2007, pp. 359-371.

${ }^{28}$ Wagner, H., "Über die Entstehung des Dynamischen Auftriebes von Tragflügeln," Zeitschrift für Angewandte Mathematik und Mechanik, Vol. 1, 1925, pp. 17-35. 
${ }^{29}$ Bisplinghoff, R. L. Ashley, H. and Halfman, R. L., Aeroelasticity, Dover Publications, Mineola, N.Y., 1996.

${ }^{30}$ Leishman, J. G., "Indicial Lift Approximations for Two-Dimensional Subsonic Flow as Obtained from Oscillatory Measurements," Journal of Aircraft, Vol. 30, No. 3, 1993, pp. 340-351.

${ }^{31}$ Singh, R. and Baeder, J., "Direct Calculation of Three-Dimensional Indicial Lift Response Using Computational Fluid Dynamics," Journal of Aircraft, Vol. 34, No. 4, 1997, pp. 465-471.

${ }^{32}$ Ghoryeshi, M., Jirásek, A., and Cummings, R. M., "Computational Investigation into the Use of Response Functions for Aerodynamic Loads Modeling," AIAA Journal, Vol. 50, No. 6, 2012, pp. 1314-1327.

${ }^{33}$ Leishman, J. G. and Crouse, G. L., "State-Space Model for Unsteady Airfoil Behavior and Dynamic Stall," AIAA Paper 89-1319, April 1989.

${ }^{34}$ Ghoreyshi, M., Badcock, K. J., and Woodgate, M. A., "Accelerating the Numerical Generation of Aerodynamic Models for Flight Simulation," Journal of Aircraft, Vol. 46, No. 3, 2009, pp. 972-980.

${ }^{35}$ Volterra, V., Theory of Functionals, Blackie, London, 1930.

${ }^{36}$ Wiener, N., Nonlinear Problems in Random Theory, MIT Press, Cambridge, MA, 1958.

${ }^{37}$ Barrett, J. F., "The Use of Functionals in the Analysis of Non-linear Physical Systems," Journal of Electr. Control, Vol. 15, 1963, pp. 567-615.

${ }^{38}$ Kalman, R. E., "Pattern Recognition Properties of Multilinear Response Functions," Journal of Control Cybern., Vol. 9, 1980, pp. 5-31.

${ }^{39}$ Stark, L., Neurological Control System: Studies in Bioengineering, Plenum Press, New York, 1968.

${ }^{40}$ Weiner, D. D. and Spina, J. F., Sinusoidal Analysis and Modeling of Weakly Nonlinear Circuits, New York: Van Nostrand Reinhold, 1980.

${ }^{41}$ Hunter, I. W. and Korenberg, M. J., "The Identification of Nonlinear Biological Systems: Wiener and Hammerstein Cascade Models," Journal of Biol. Cybern., Vol. 55, 1986, pp. 135-144.

${ }^{42}$ Korenberg, M. J., "A Fast Orthogonal Search Method For Biological Time-Series Analysis and System Identification," Proceeding of IEEE International Conference Sys. Man. Cybernet., 1989, pp. 459-465.

${ }^{43}$ Korenberg, M. J., "A Robust Orthogonal For System Identification and Time-Series Analysis," Journal of Biological Cybernet, Vol. 60, 1989, pp. 267-276.

${ }^{44}$ Jirásek, A. and Cummings, R. M., "Application of Volterra Functions to X-31 Aircraft Model Motion," AIAA Paper 2009-3629, June 2009.

${ }^{45}$ Marques, F. D. and Anderson, J., "Identification and Prediction of Unsteady Transonic Aerodynamic Loads by MultiLayer Functionals," Journal of Fluids and Structure, Vol. 1, No. 6, 2011, pp. 83-106.

${ }^{46}$ Silva, W. A., "Discrete-Time Linear and Nonlinear Aerodynamic Impulse Responses for Efficient CFD Analysis," PhD Dissertation, Faculty of the Department of Applied Science, The College of William and Mary, Williamsburg, VA, September 1997.

${ }^{47}$ Da Ronch, A., McCracken, A., Badcock, K. J., Ghoreyshi, M., and Cummings, R. M., "Modeling of Unsteady Aerodynamic Loads," AIAA Paper 2011-2376, Aug. 2011.

${ }^{48}$ Faller, W. E. and Schreck, S. J., "Neural Networks: Applications and Opportunities in Aeronautics," Progress in Aerospace Sciences, Vol. 32, No. 5, 1996, pp. 433-456.

${ }^{49}$ Faller, W. E. and Schreck, S. J., "Real-Time Prediction of Unsteady Aerodynamics: Application for Aircraft Control and Maneuverability Enhancement," IEEE Trans. Neural Networks, Vol. 6, No. 6, 1995, pp. 1461-1468.

${ }^{50}$ Glaz, B., Liu, L., and Friedmann, P. P., "Reduced-Order Nonlinear Unsteady Aerodynamic Modeling Using a SurrogateBased Recurrence Framework," AIAA Journal, Vol. 48, No. 10, 2010, pp. 2418-2429.

${ }^{51}$ Ghoryeshi, M., Jirásek, A., Post, M. L., and Cummings, R. M., "Computational Approximation of Nonlinear Aerodynamics Using an Aerodynamic Model Hierachy," AIAA Paper 2011-3667, June 2011.

${ }^{52}$ Strang, W. Z., Tomaro, R. F., and Grismer, M. J., "The Defining Methods of Cobalt: A Parallel, Implicit, Unstructured Euler/Navier-Stokes Flow Solver," AIAA Paper 1999-0786, 1999.

${ }^{53}$ Da Ronch, A., Vallespin, D., Ghoreyshi, M., and Badcock, K. J., "Evaluation of Dynamic Derivatives Using Computational Fluid Dynamics," AIAA Journal, Vol. 50, No. 2, 2012, pp. 470-484.

${ }^{54}$ Gottlieb, J. J. and Groth, C. P. T., "Assessment of Riemann Solvers For Unsteady One-dimensional Inviscid Flows of Perfect Gasses," Journal of Fluids and Structure, Vol. 78, No. 2, 1998, pp. 437-458.

${ }^{55}$ Tomaro, R. F., Strang, W. Z., and Sankar, L. N., "An Implicit Algorithm For Solving Time Dependent Flows on Unstructured Grids," AIAA Paper 1997-0333, 1997.

${ }^{56}$ Forsythe, J. R., Hoffmann, K. A., Cummings, R. M., and Squires, K. D., "Detached-Eddy Simulation With Compressibility Corrections Applied to a Supersonic Axisymmetric Base," Journal of Fluids Engineering, Vol. 124, No. 4, 2002, pp. 911-923.

${ }^{57}$ Forsythe, J. R. and Woodson, S. H., "Unsteady Computations of Abrupt Wing Stall Using Detached-Eddy Simulations," Journal of Aircraft, Vol. 42, No. 3, 2005, pp. 606-616.

${ }^{58}$ Morton, S. A., Forsythe, J. R., Mitchell, A. M., and Hajek, D., "Detached-Eddy Simulations and Reynolds-Averaged Navier-Stokes Simulations of Delta Wing Vortical Flowfields," Journal of Fluids Engineering, Vol. 124, No. 4, 2002 , pp. 924-932.

${ }^{59}$ Forsythe, R., Squires, K. D., Wurtzler, K. E., and Spalart, P. R., "Detached-Eddy Simulation of Fighter Aircraft at High Alpha," Journal of Aircraft, Vol. 41, No. 2, 2004, pp. 193-200.

${ }^{60}$ Jeans, T., McDaniel, D., Cummings, R. M., and Mason, W., "Aerodynamic Analysis of a Generic Fighter Using Delayed Detached-Eddy Simulations," Journal of Aircraft, Vol. 46, No. 4, 2009, pp. 1326-1339.

${ }^{61}$ Balajewicz, M., Nitzsche, F., and Feszty, D., "Application of Multi-Input Volterra Theory to Nonlinear Multi-Degree-ofFreedom Aerodynamic Systems," AIAA Journal, Vol. 48, No. 1, 2010, pp. 56-62.

${ }^{62}$ Duffy, D. G., Advanced Engineering Mathematics With MATLAB, second edition, Chapman and Hall/CRC, Florida, 2003. 
${ }^{63}$ Romero, D. A., Amon, C., Finger, S., and Verdinelli, I., "Multi-Stage Bayesian Surrogates For the Design of TimeDependent System," Proceedings of ASME 2004 Design Engineering Technical Conference and Computers and Information in Engineering, DETC 2004-57510, 2004.

${ }^{64}$ Jones, D. R., Schonlau, M., and Welch, W. J., "Efficient Global Optimization of Expensive Black-Box Functions," Kluwer Academic Publications, Vol. 13, 1998, pp. 455-492.

${ }^{65}$ Da Ronch, A., Ghoreyshi, M., and Badcock, K. J., "On the Generation of Flight Dynamics Aerodynamic Tables by Computational Fluid Dynamics," Progress in Aerospace Sciences, Vol. 47, 2011, pp. 597-620.

${ }^{66}$ Arbib, M. A., The Handbook of Brain Theory and Neural Networks, Second Edition, The MIT Press, 2003.

${ }^{67}$ Dasgupta, D. and Michalewicz, Z., Evolutionary Algorithms in Engineering Applications, Springer-Verlag, Berlin, Heidelberg, 1997.

${ }^{68}$ Balajewicz, M., Nitzsche, F., and Feszty, D., "Reduced Order Modeling of Nonlinear Transonic Aerodynamics Using a Pruned Volterra Series," 50th AIAA/ASME/ASCE/AHS/ASC Structures, Structural Dynamics and Materials Conference, Palm Springs, CA 2009

${ }^{69}$ Morelli, E. A., "System IDentification Programs for AirCraft (SIDPAC)," AIAA Paper 2002-4704, August 2002.

${ }^{70}$ Görtz, S., McDaniel, D., and Morton, S. A., "Towards an Efficient Aircraft Stability and Control Analysis Capability Using High-Fidelity CFD," AIAA Paper 2007-1053, Jan 2007.

${ }^{71}$ O'Neill, C. and Arena, A., "Time Domain Training Signals Comparison for Computational Fluid Dynamics Based Aerodynamic Identification," Journal of Aircraft, Vol. 42, No. 2, 2005, pp. 421.428.

${ }^{72}$ Murpy, P. C. and Klein, V., "Validation of Methodology for Estimating Aircraft Unsteady Aerodynamics Parameters from Dynamic Wind Tunnel Tests," AIAA Paper 2003-5397, August 2003.

${ }^{73}$ Morelli, E. A., "Real Time Parameters Estimation in the Frequency Domain," AIAA Paper 99-4043, August 1999.

${ }^{74}$ Cummings, R. M. and Schütte, A., "An Integrated Computational/Experimental Approach to UCAV Stability \& Control Estimation: Overview of NATO RTO AVT-161," AIAA Paper 2010-4392, June-July 2010.

${ }^{75}$ Canter, D. E. and Groves, A. W., "X-31 Post-Stall Envelope Expansion and Tactical Utility Testing," AIAA Paper 1994-2171, June 1994.

${ }^{76}$ Alcorn, C. W., Croom, M. A., and Francis, M. S., "The X-31 Experience - Aerodynamic Impediments to Post-Stall Agility," AIAA Paper 1995-362, Jan 1995.

${ }^{77}$ Williams, D. L., Nelson, R. C., and Fisher, D., "An Investigation of X-31 Roll Characteristics at High Angle-of-Attack Through Subscale Model Testing," AIAA Paper 1994-806, Jan 1994.

${ }^{78}$ Rein, M., Höhler, G., Schütte, A., Bergmann, A., and Löser, T., "Ground-Based Simulation of Complex Maneuvers of a Delta-Wing Aircraft," AIAA Paper 2006-3149, June 2006.

${ }^{79}$ Schefter, J., "X-31: How They're Inventing a Radical New Way to Fly," Popular Science, Vol. 234, No. 2, 1989, pp. 58-64.

${ }^{80}$ Tyssel, L., "Hybrid Grid Generation for Complex 3D Geometries," Proceedings of the 7th International Conference on Numerical Grid Generation in Computational Field Simulation, 2000, pp. 337-346.

${ }^{81}$ Tyssel, L., "The TRITET Grid Generation System," International Society of Grid Generation (ISGG)," Proceedings of the 10th International Conference on Numerical Grid Generation in Computational Field Simulations, Forth, Crete, Greece, 2000 .

${ }^{82}$ Spalart, P. R. and Allmaras, S. R., "A One Equation Turbulence Model for Aerodynamic Flows,” AIAA Paper 1992-0439, January 1992

${ }^{83}$ Spalart, P. R. and Schur, M., "On the Sensitisation of Turbulence Models to Rotation and Curvature," Aerospace Science and Technology, Vol. 1, 1997, pp. 297-302.

${ }^{84}$ Menter, F., "Two-Equation Eddy-Viscosity Turbulence Models for Engineering Applications," AIAA Journal, Vol. 32, No. 8, 1994, pp. 1598-1605.

${ }^{85}$ Mackman, T. J., Allen, C. B., Ghoreyshi, M., and Badcock, K. J., "Comparison of Adaptive Sampling Methods for Generation of Aerodynamic Models for Flight Simulations," AIAA Paper 2011-1171, January 2011.

${ }^{86}$ Leishman, J. G., "Subsonic Unsteady Aerodynamics Caused by Gusts Using the Indicial Method," Journal of Aircraft, Vol. 33, No. 5, 1996, pp. 869-879. 


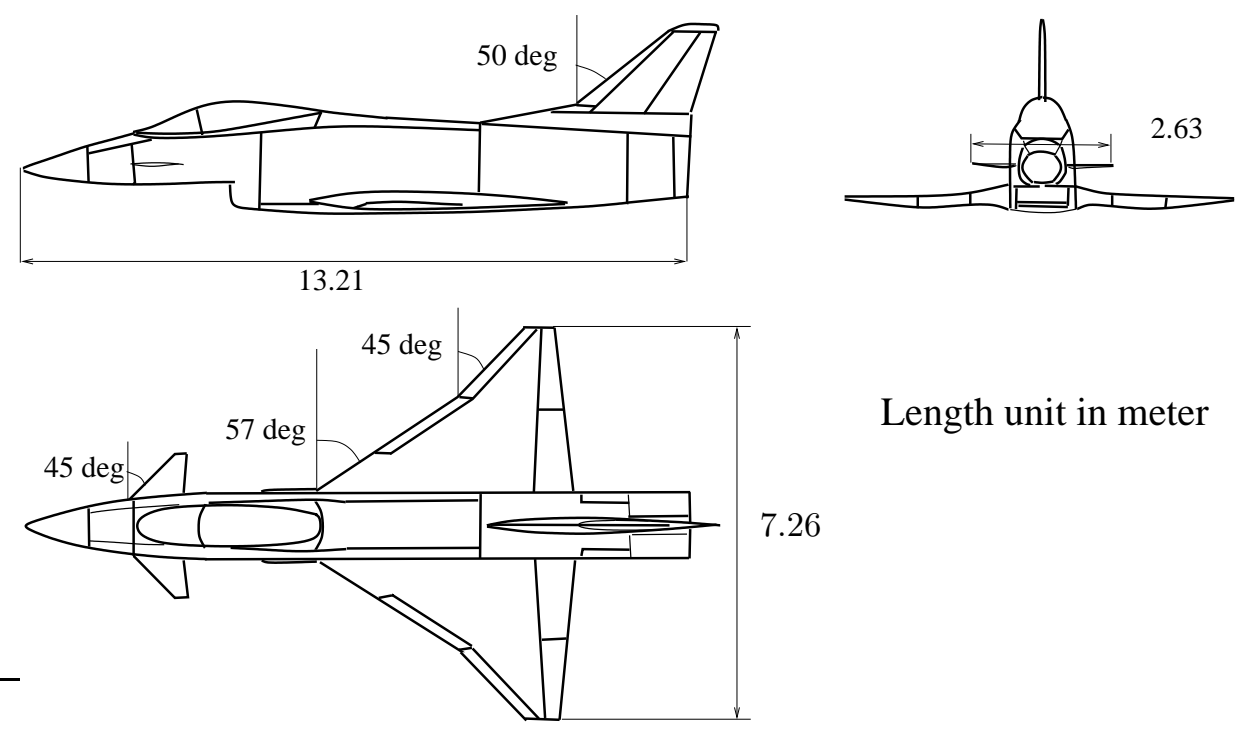

Figure 1. The X-31 aircraft geometry

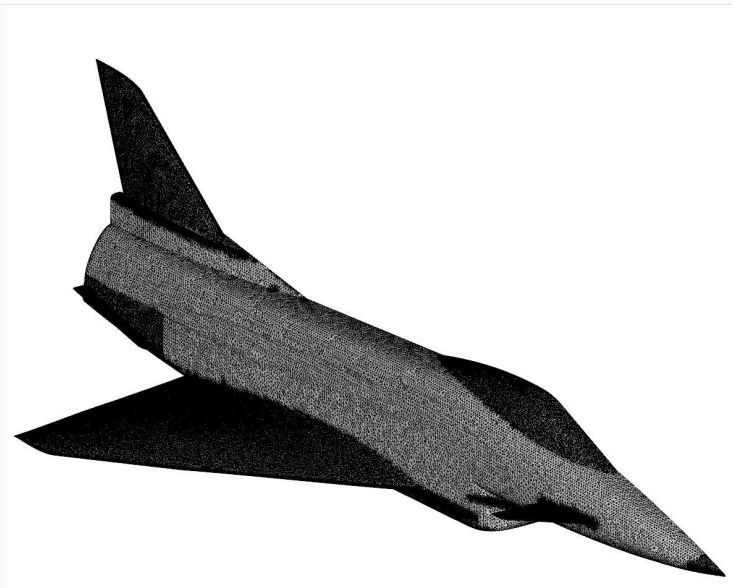

(a) Half-Model Mesh

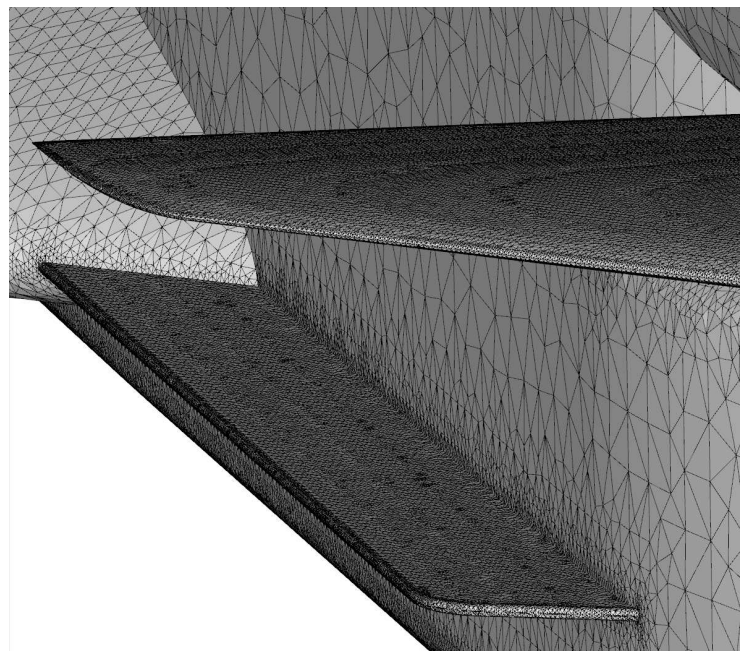

(b) Surface mesh around LEX and canard

Figure 2. The X-31 aircraft mesh model 


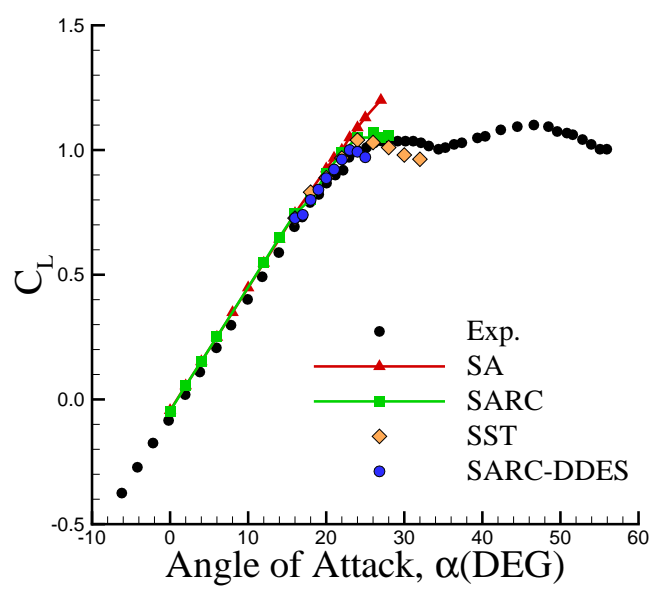

(a) lift coefficient

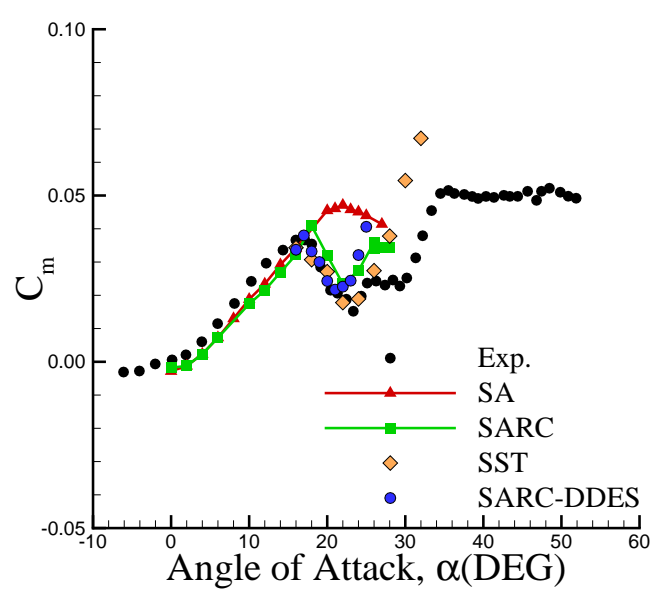

(c) pitching moment coefficient

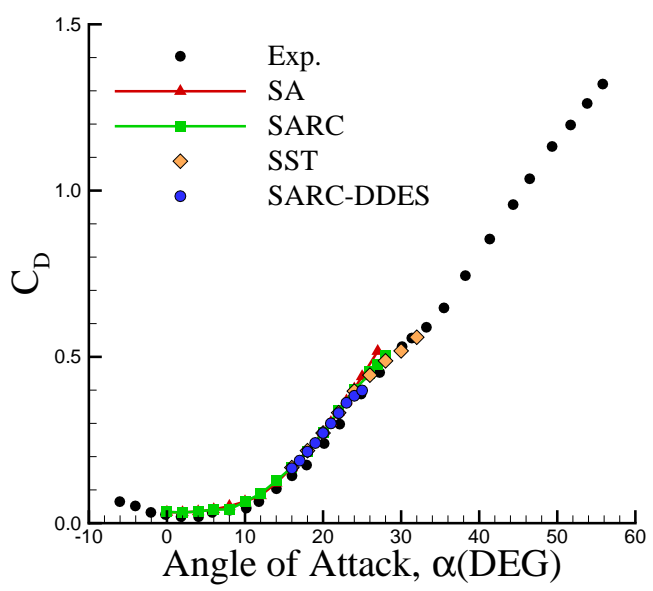

(b) drag coefficient

Figure 3. The X-31 static loads validations. The static conditions are : $M_{\infty}=0.18$ and $R e=2 \times 10^{6}$. 


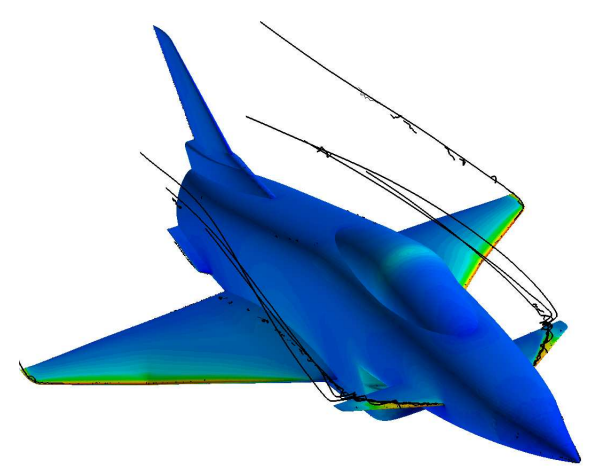

(a) $\alpha=10^{0}$

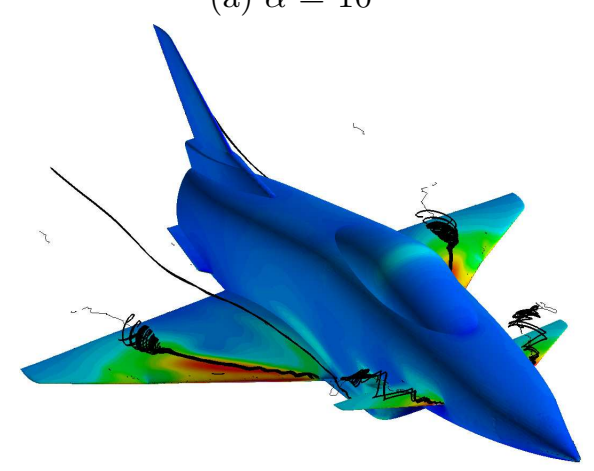

(c) $\alpha=18^{0}$

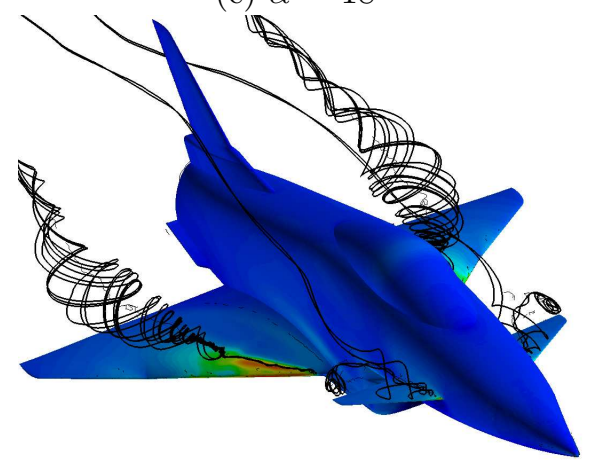

(e) $\alpha=22^{0}$

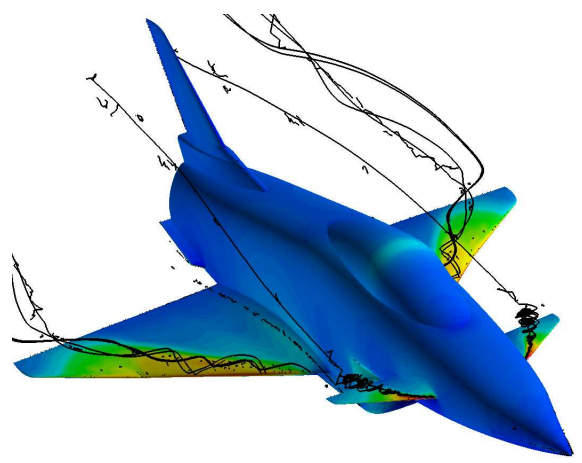

(b) $\alpha=14^{0}$

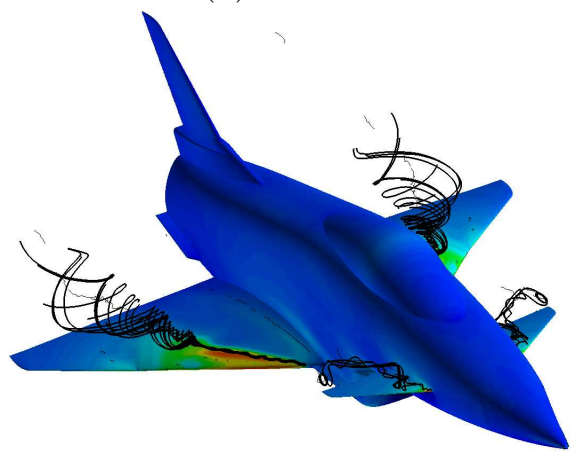

(d) $\alpha=20^{0}$

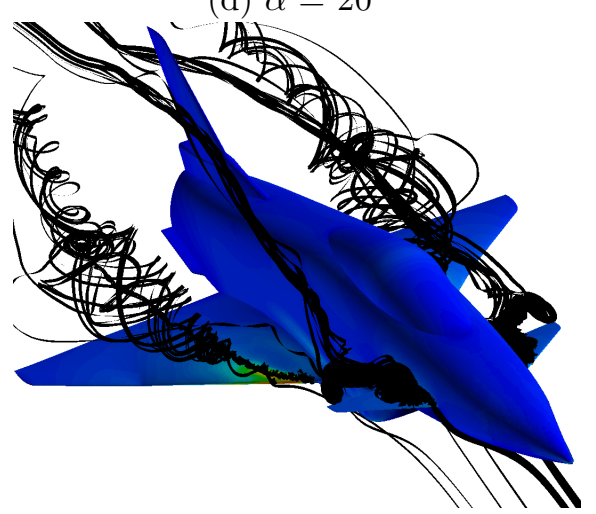

(f) $\alpha=25^{0}$

Figure 4. The X-31 vortical flows using DDES-SARC turbulence model. The conditions are $: M_{\infty}=0.18$ and $R e=2 \times 10^{6}$. 


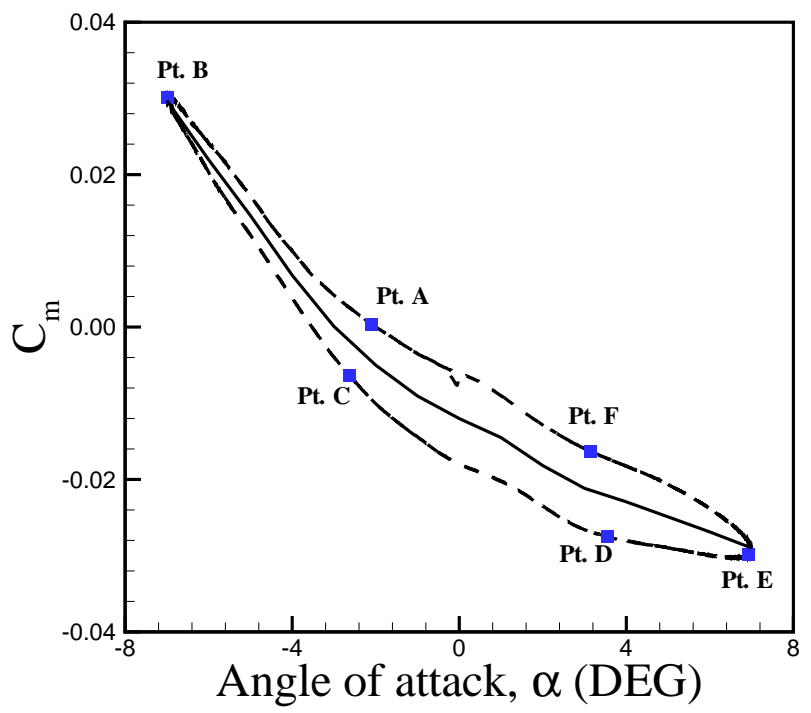

(a) Target motion.

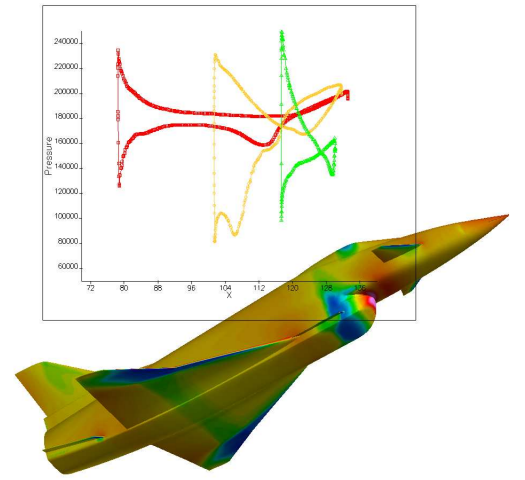

(b) Point A

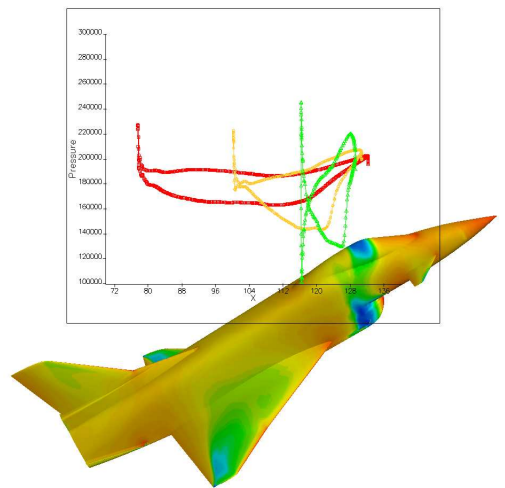

(e) Point D

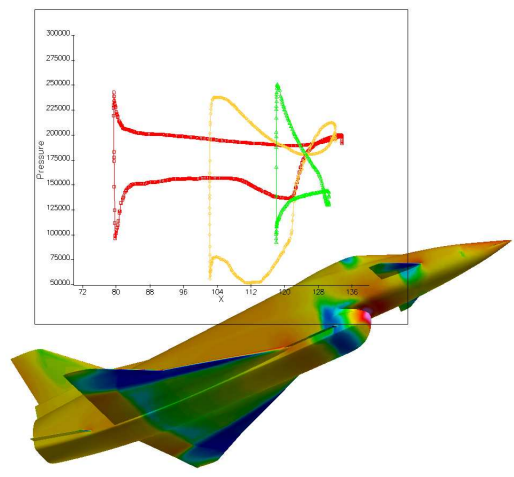

(c) Point B

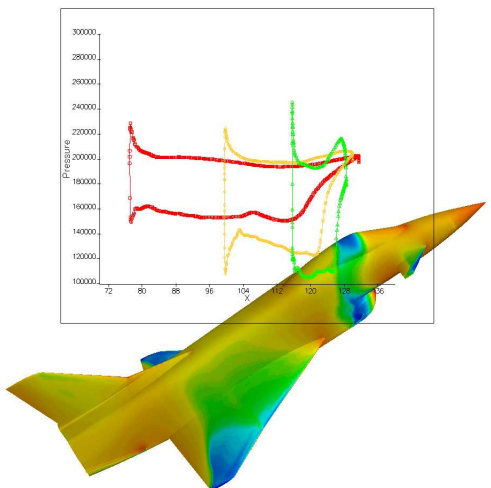

(f) Point E

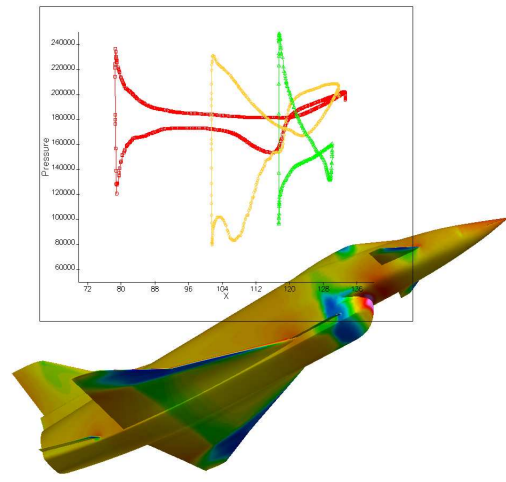

(d) Point C

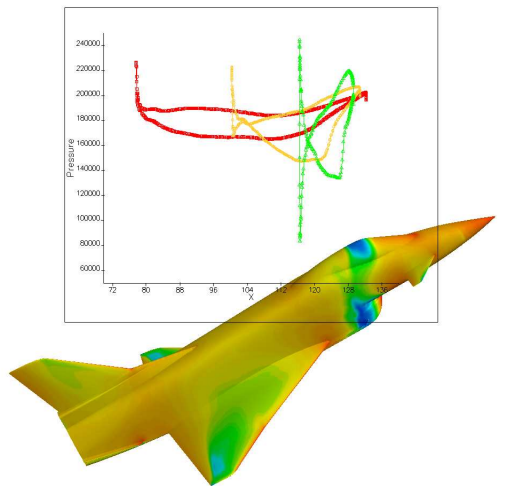

(g) Point F

Figure 5. Target motion is a pitch harmonic motion as: $\alpha=7^{\circ} \sin (\omega t), M_{\infty}=\mathbf{0 . 9}, k=0.01$, and $R e=2 \times 10^{6}$. In Fig. (a) static data are shown with a solid line. 


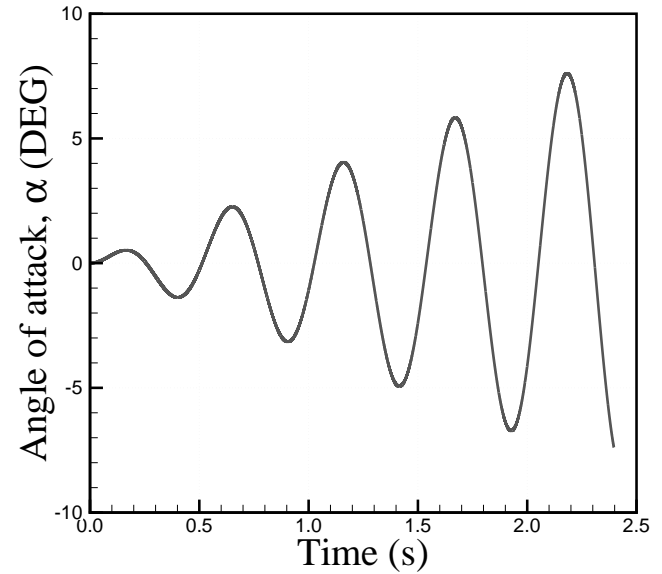

(a) Spiral maneuver

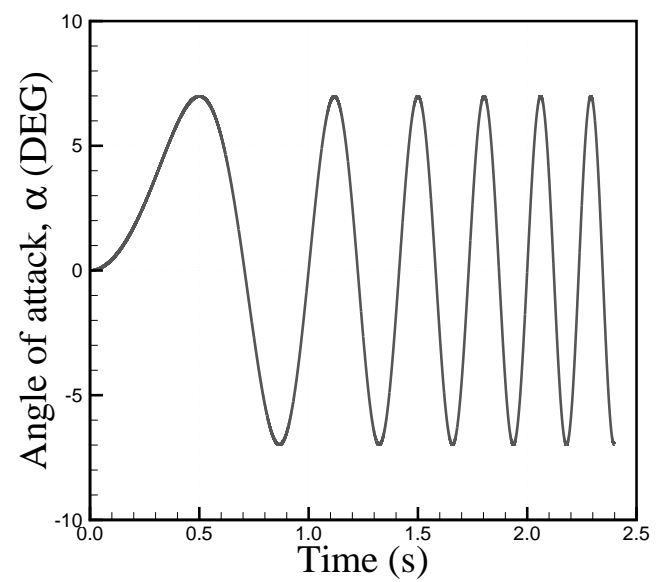

(c) Chirp maneuver

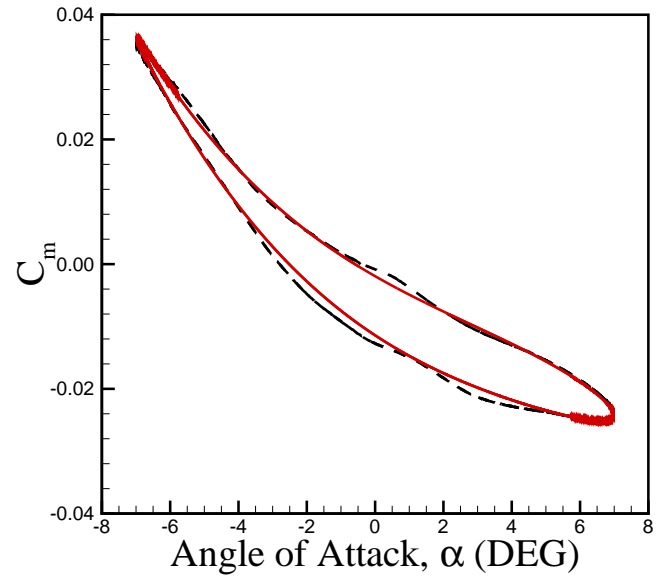

(b) ROM prediction

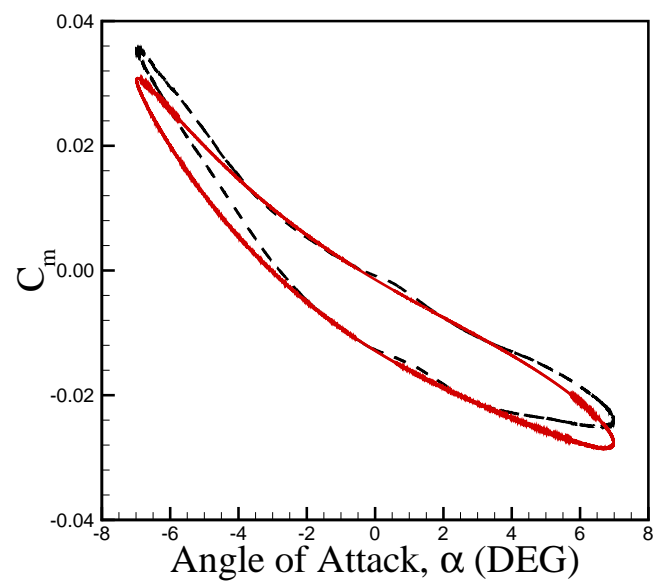

(d) ROM prediction

Figure 6. Volterra reduced order modeling using Spiral and Chirp training maneuvers. The flow conditions of training maneuvers are : $M_{\infty}=0.9$ and $R e=2 \times 10^{6}$. 


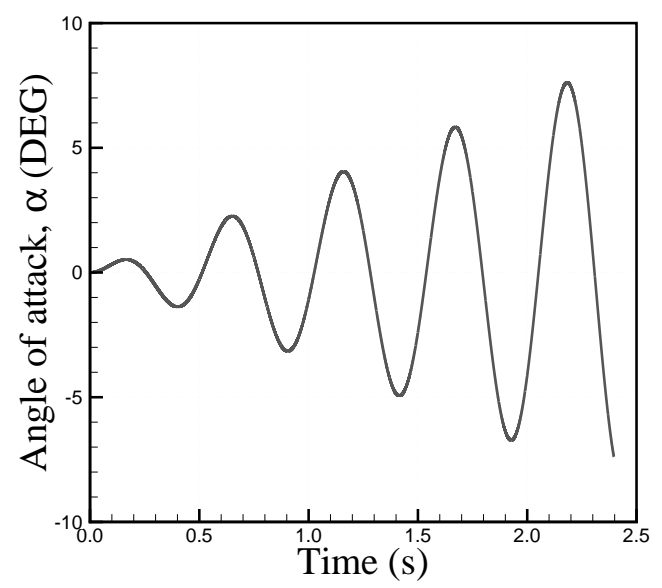

(a) Spiral maneuver

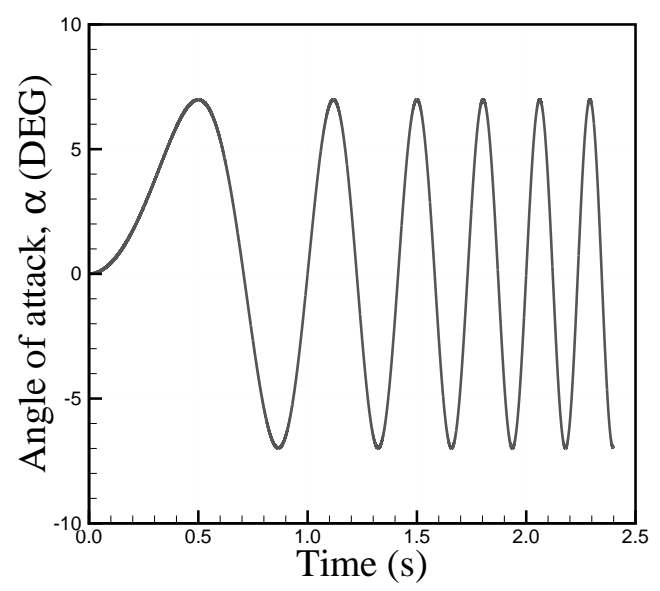

(c) Chirp maneuver

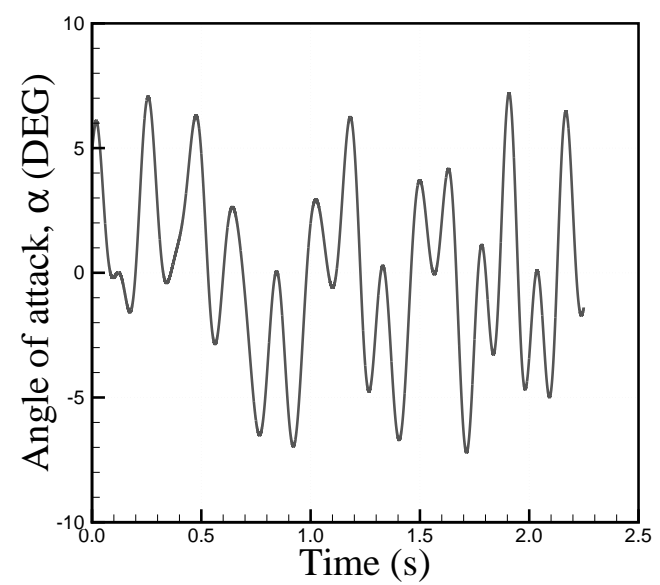

(e) Schroeder maneuver

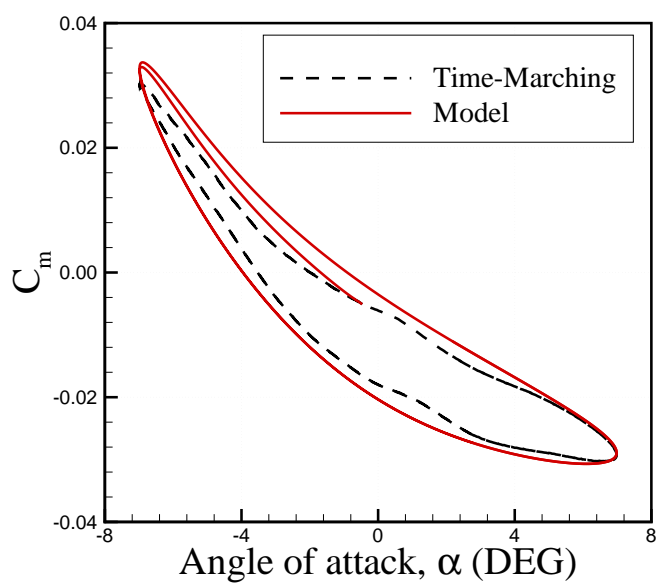

(b) ROM prediction

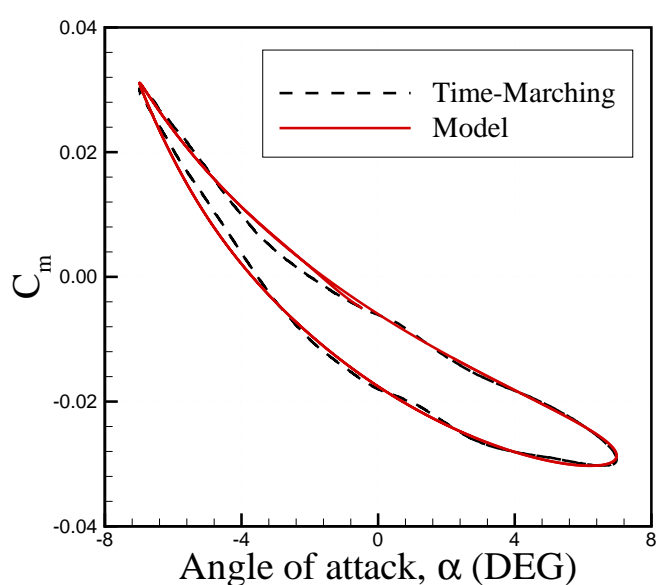

(d) ROM prediction

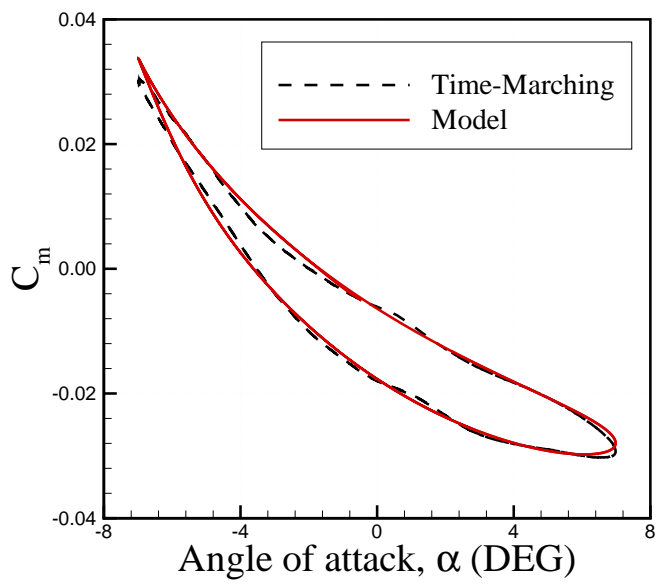

(f) ROM prediction

Figure 7. RBF reduced order modeling- Training maneuver are a Spiral, a Chirp, and a Schroeder motion. The flow conditions are : $M_{\infty}=\mathbf{0 . 9}$ and $R e=2 \times 10^{6}$. 


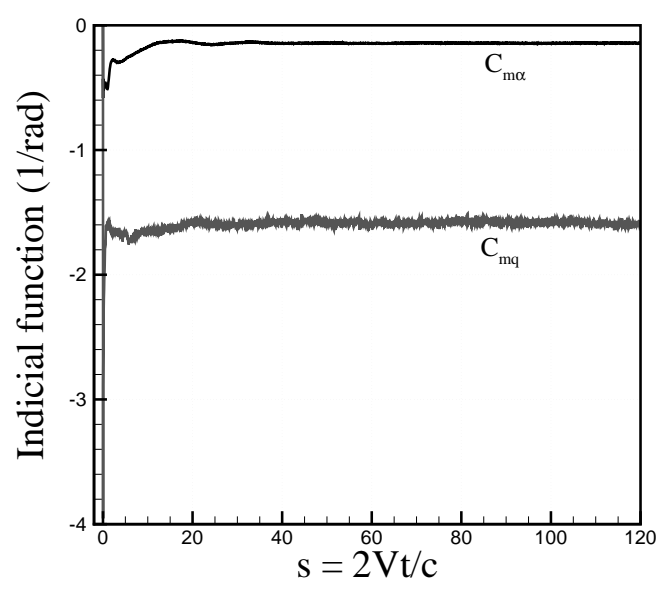

(a) linear responses

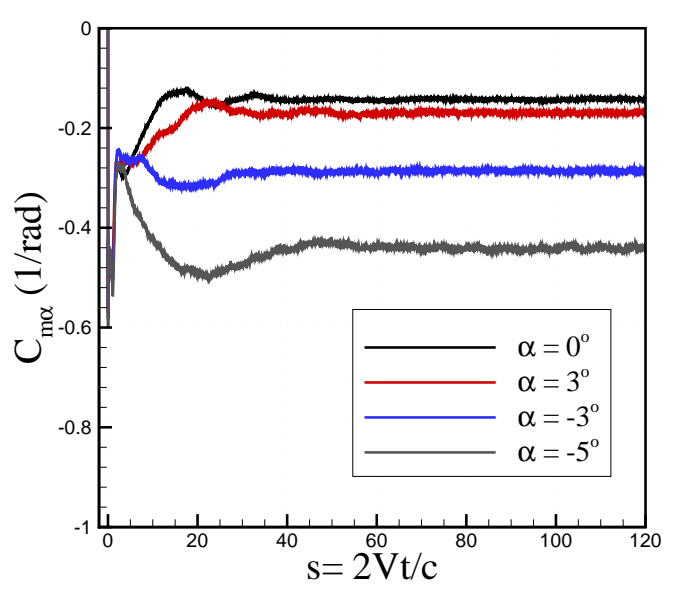

(c) non-linear responses

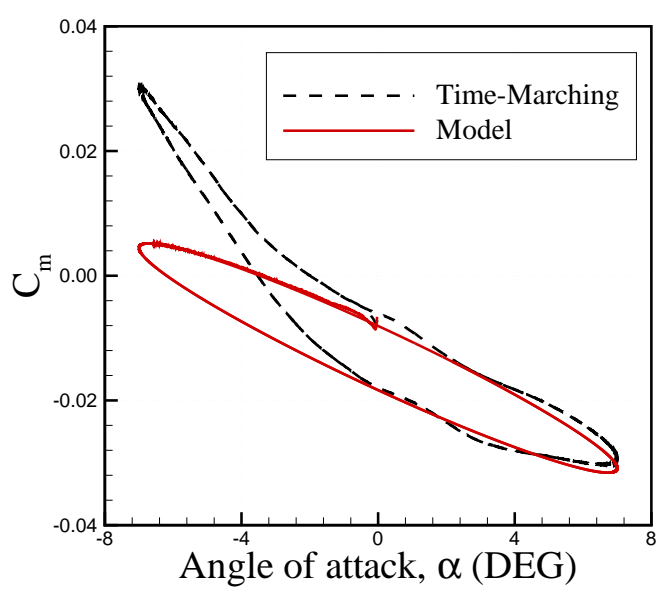

(b) ROM based on linear responses

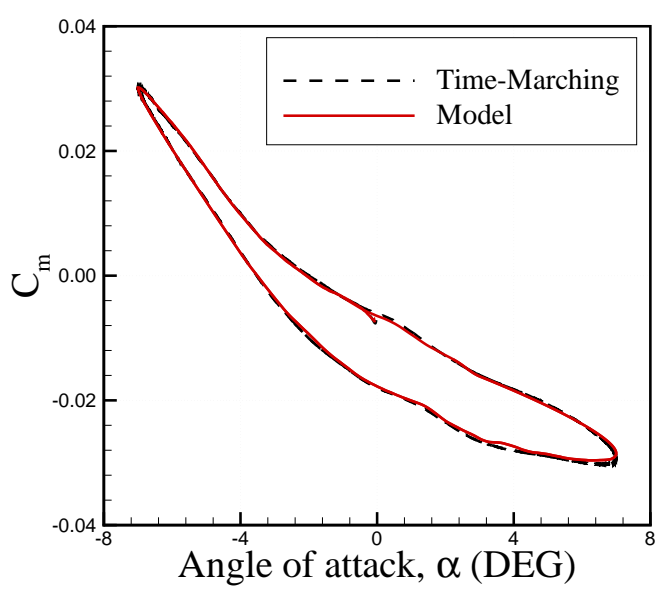

(d) ROM based on non-linear responses

Figure 8. ROM using indicial functions. The flow conditions are : $M_{\infty}=\mathbf{0 . 9}$ and $R e=2 \times 10^{6}$. 


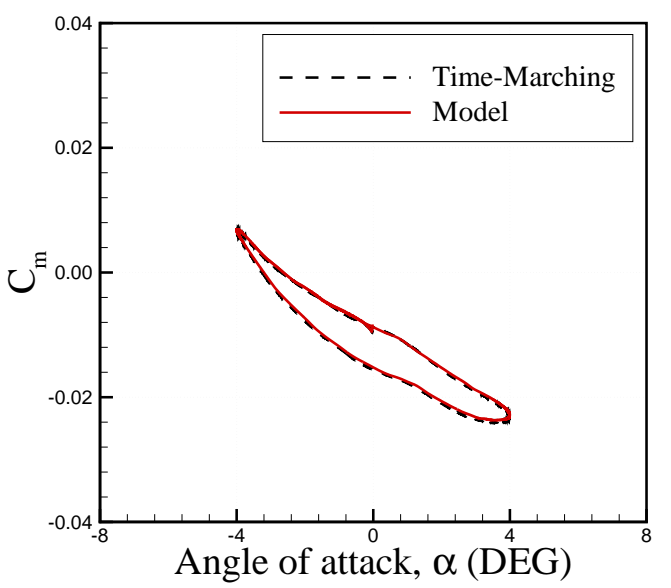

(a) $\alpha=4^{\circ} \sin (\omega t), k=0.01$

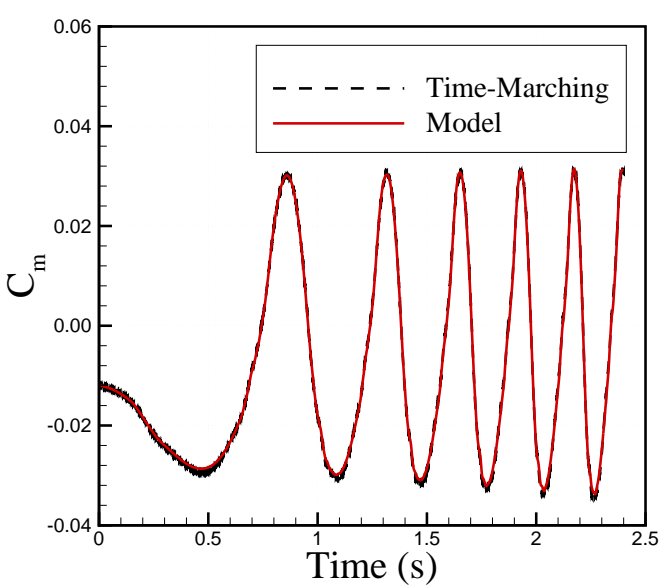

(c) chirp maneuver

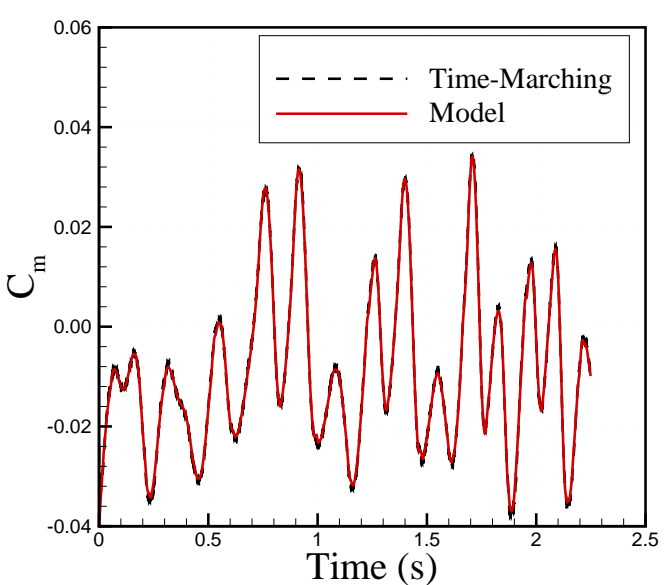

(e) schroder maneuver

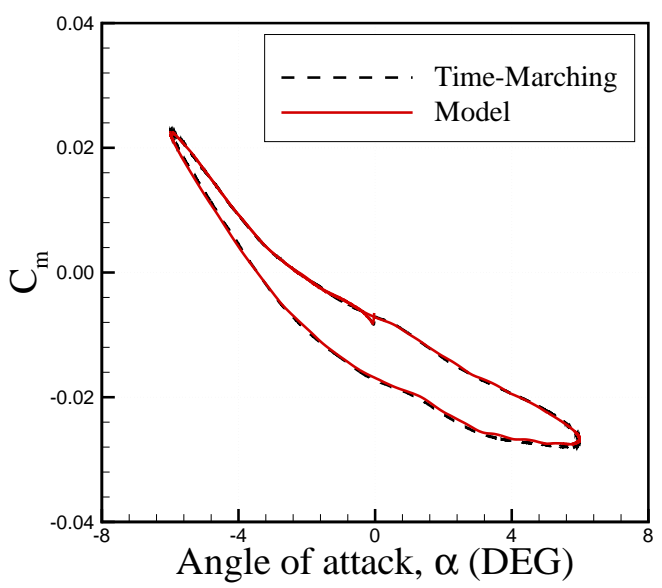

(b) $\alpha=6^{\circ} \sin (\omega t), k=0.01$

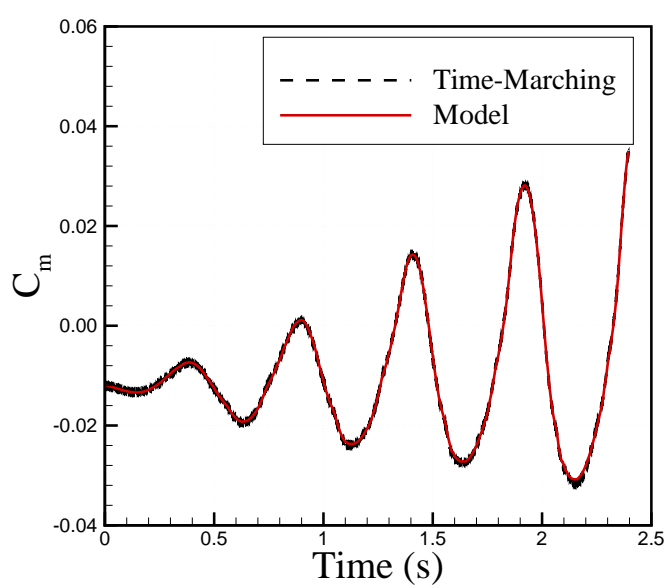

(d) spiral maneuver

The flow conditions are : $M_{\infty}=0.9$ and $R e=2 \times 10^{6}$. 


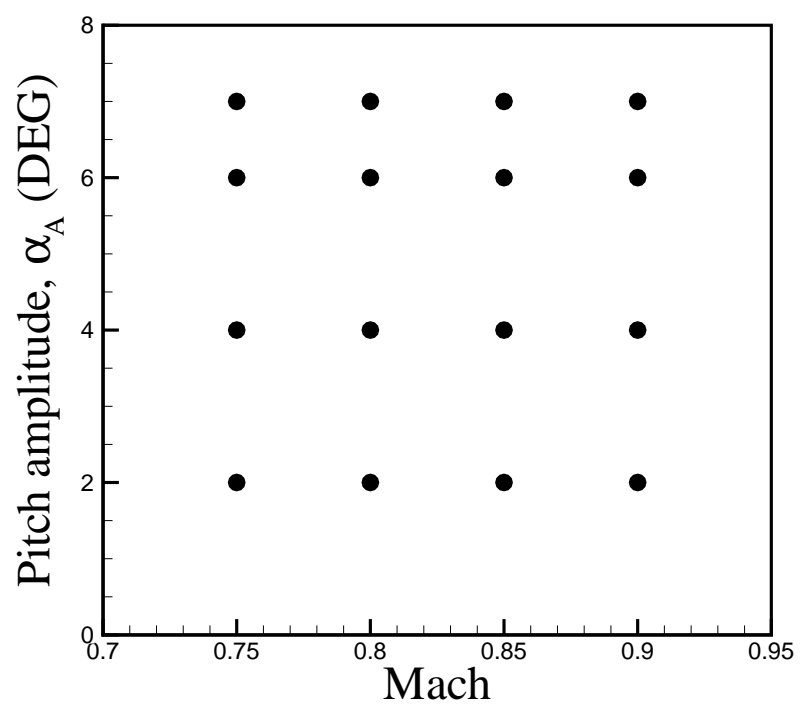

(a) Samples Design for SBRF model.

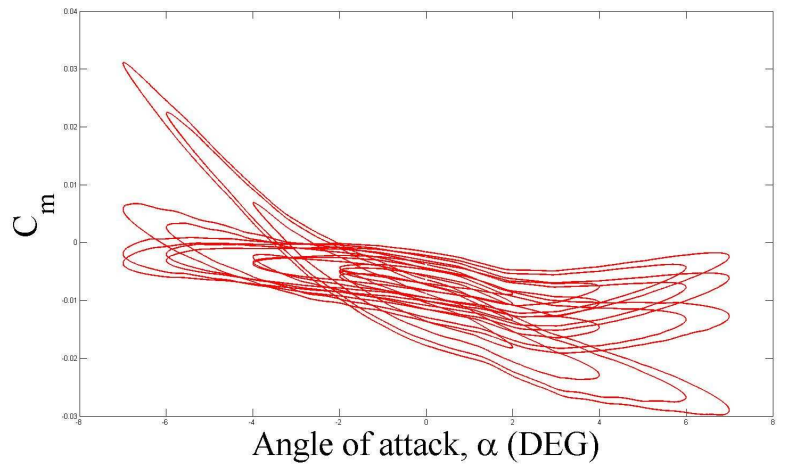

(a) Training pitch motions for SBRF model.

Figure 10. Samples and training pitch motions for a SBRF model. 


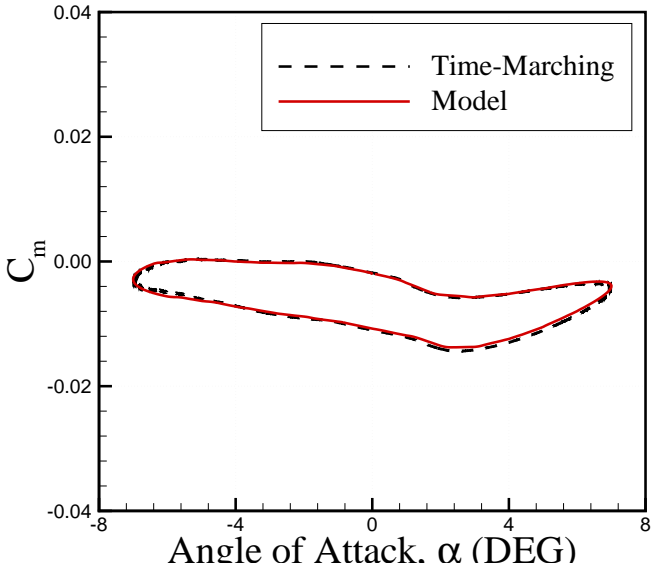

Angle of Attack, $\alpha$ (DEG)

(a) $\alpha=7^{\circ} \sin (\omega t), k=0.01, \mathrm{M}=0.78$

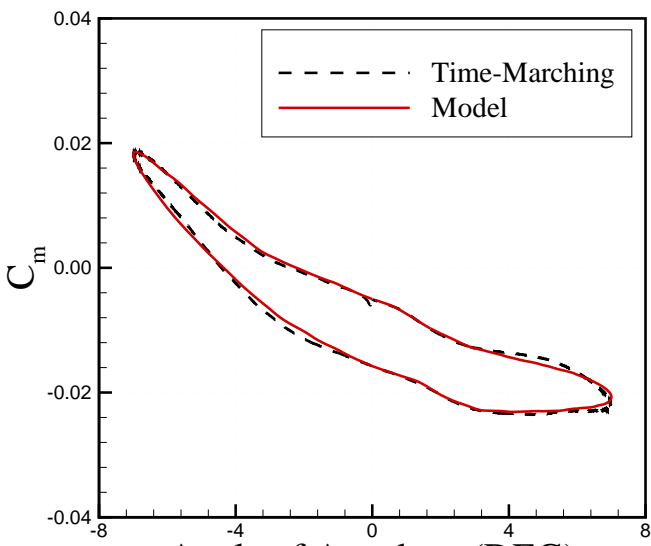

Angle of Attack, $\alpha$ (DEG)

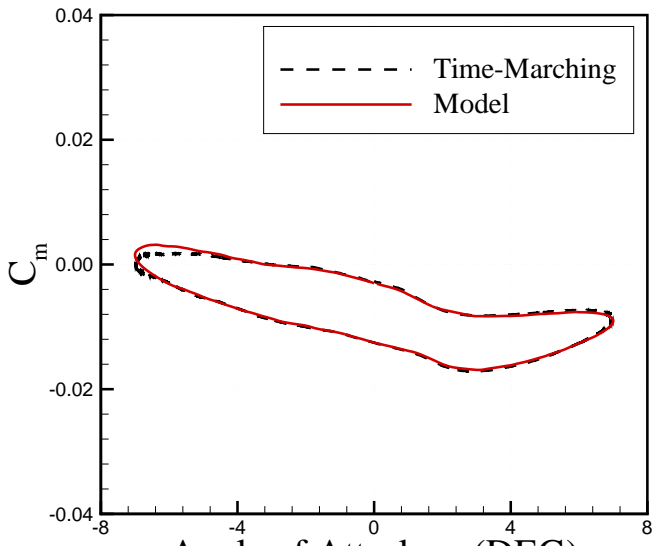

Angle of Attack, $\alpha$ (DEG)

(b) $\alpha=7^{\circ} \sin (\omega t), k=0.01, \mathrm{M}=0.825$

(e) $\alpha=7^{\circ} \sin (\omega t), k=0.01, \mathrm{M}=0.88$

Figure 11. Transonic loads modeling in Mach number/angle of attack space at fixed reduced frequency. The ROM is a SBRF model. In above $\omega$ is angular velocity and $k=\omega c / 2 V$ is reduced frequency. 


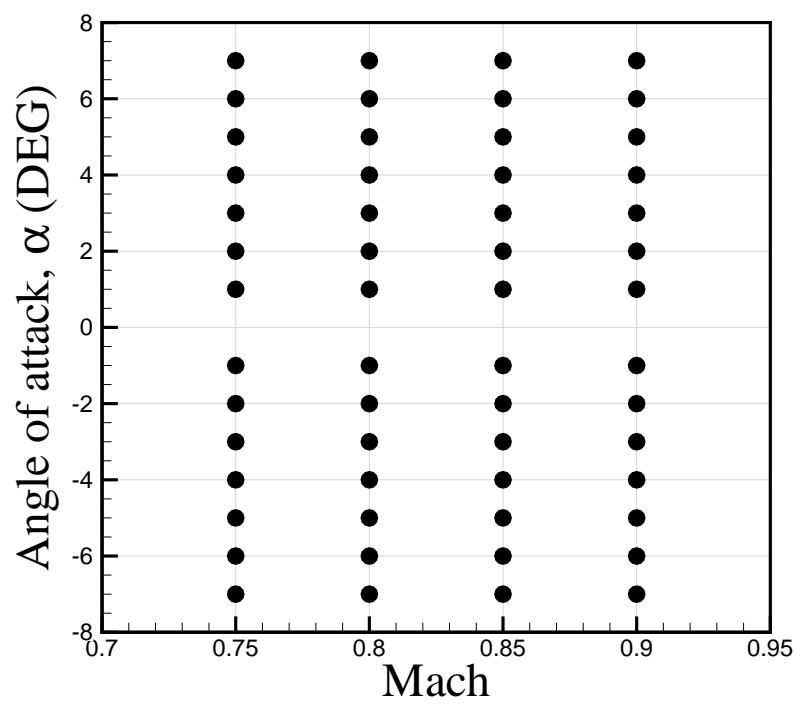

(a) Samples Design for indicial functions.

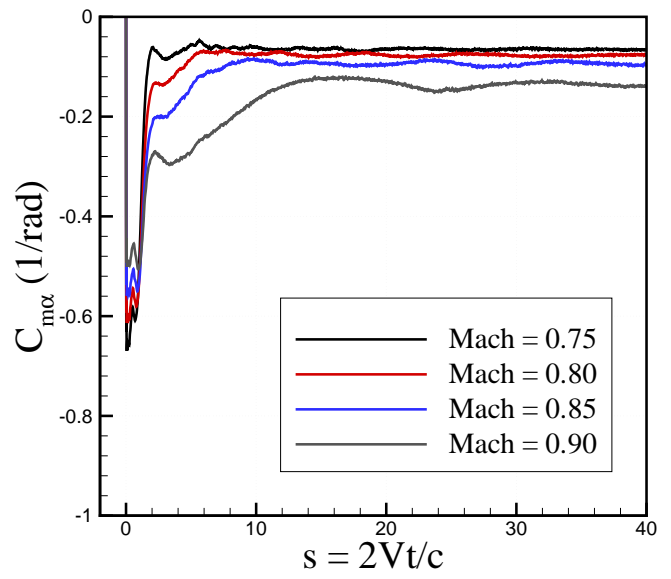

(a) Angle of attack indicial functions

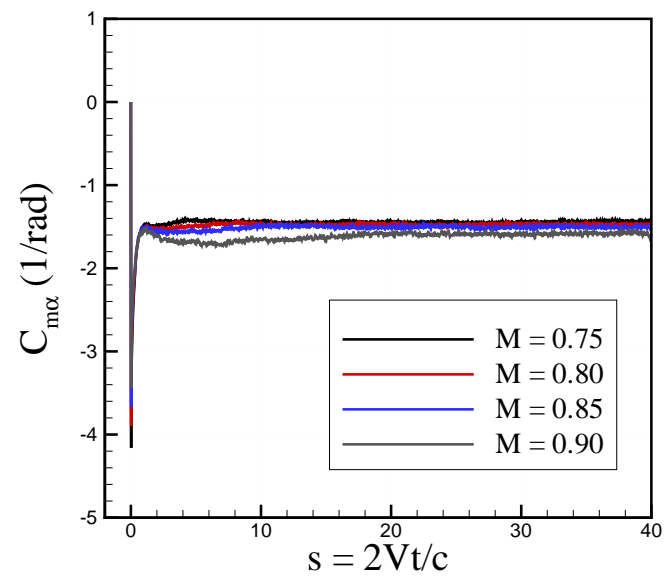

(a) Pitch rate indicial functions

Figure 12. Time-dependent surrogate modeling of indicial functions. 


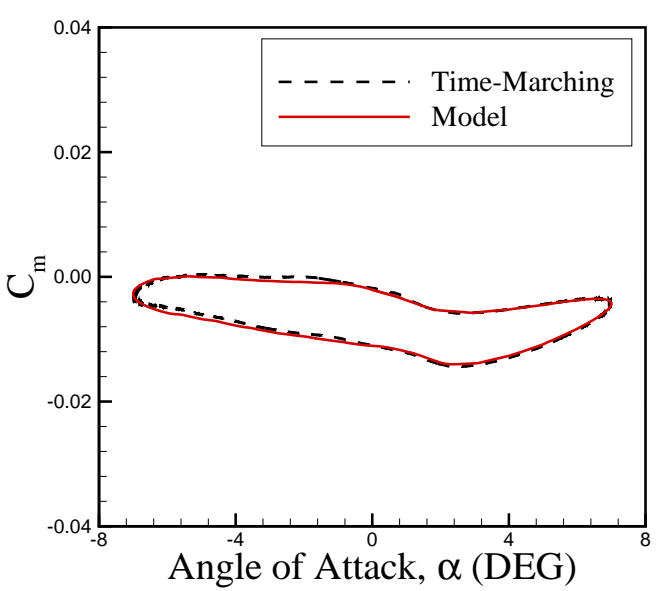

(a) $\alpha=7^{\circ} \sin (\omega t), k=0.01, \mathrm{M}=0.78$

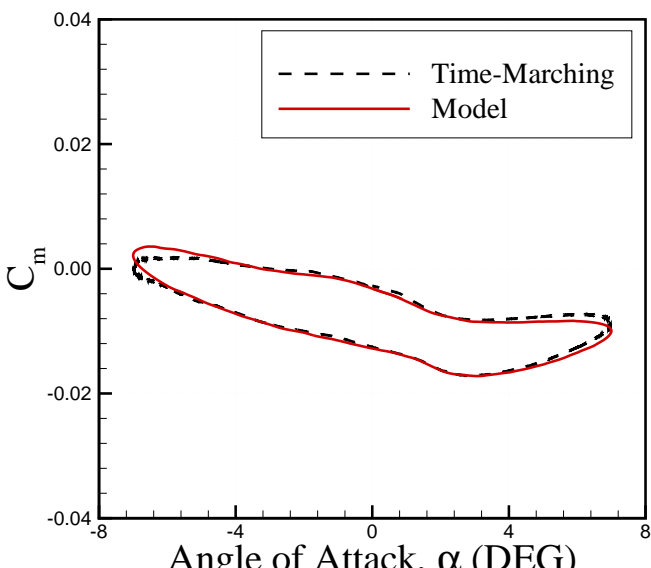

Angle of Attack, $\alpha$ (DEG)

(c) $\alpha=7^{\circ} \sin (\omega t), k=0.01, M=0.825$

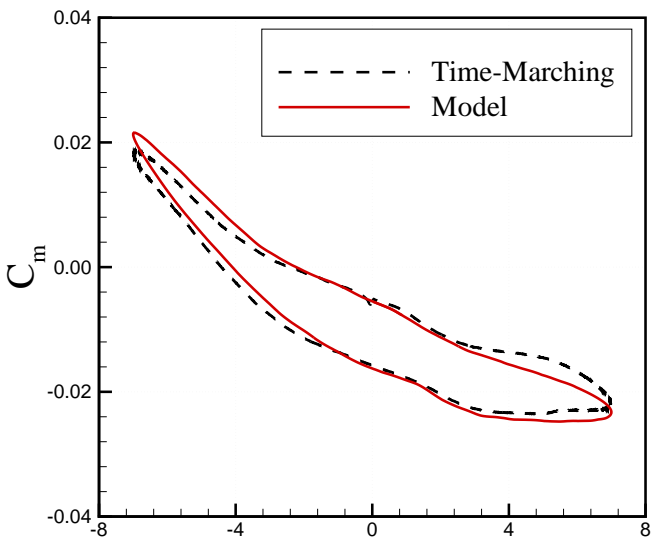

Angle of Attack, $\alpha$ (DEG)

(e) $\alpha=7^{\circ} \sin (\omega t), k=0.01, \mathrm{M}=0.88$

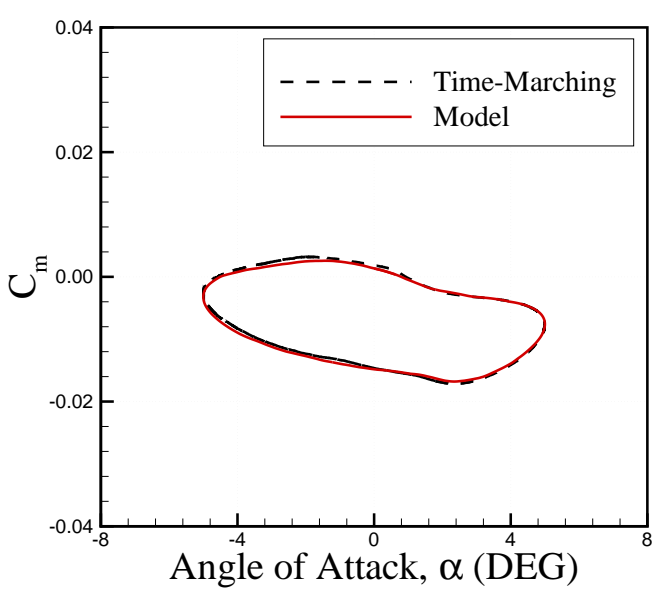

(b) $\alpha=5^{\circ} \sin (\omega t), k=0.025, \mathrm{M}=0.78$

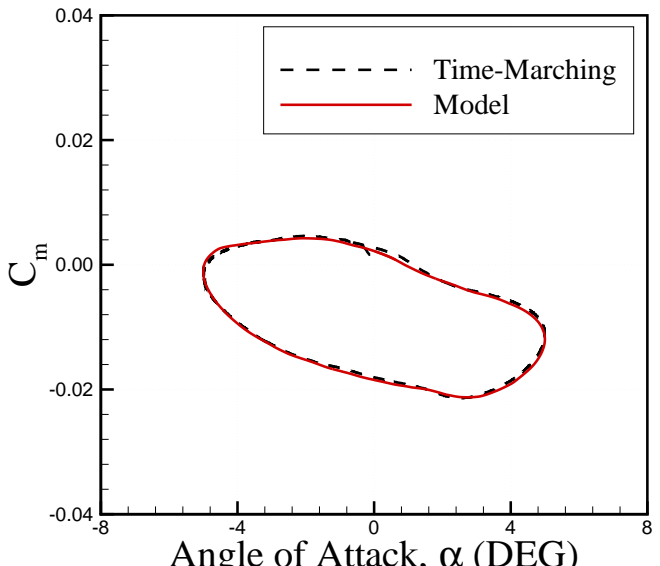

(d) $\alpha=5^{\circ} \sin (\omega t), k=0.03, M=0.825$

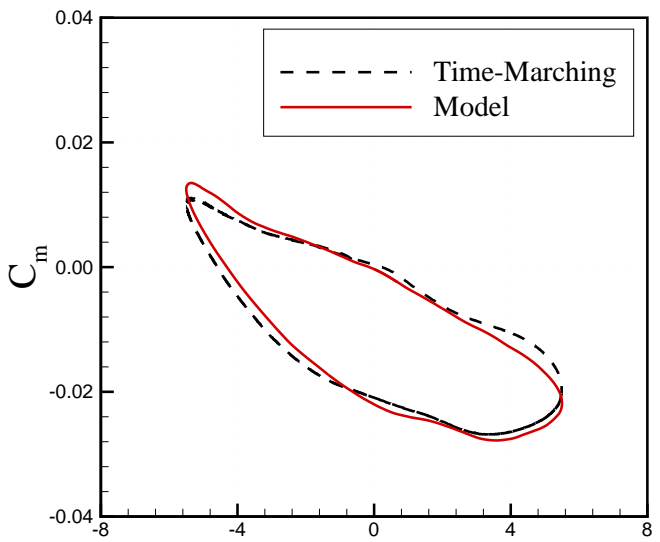

Angle of Attack, $\alpha$ (DEG)

(f) $\alpha=5.5^{\circ} \sin (\omega t), k=0.025, \mathrm{M}=0.88$

Figure 13. Transonic loads modeling in Mach number/angle of attack/frequency space. The ROM is based on a time-dependent surrogate model that approximates the non-linear indicial functions at different flight conditions. In above $\omega$ is angular velocity and $k=\omega c / 2 V$ is reduced frequency. 